\title{
THE DIRECT SCATTERING PROBLEM OF OBLIQUELY INCIDENT ELECTROMAGNETIC WAVES BY A PENETRABLE HOMOGENEOUS CYLINDER
}

\author{
DROSSOS GINTIDES AND LEONIDAS MINDRINOS \\ Communicated by Fioralba Cakoni
}

\begin{abstract}
In this paper we consider the direct scattering problem of obliquely incident time-harmonic electromagnetic plane waves by an infinitely long dielectric cylinder. We assume that the cylinder and the outer medium are homogeneous and isotropic. From the symmetry of the problem, Maxwell's equations are reduced to a system of two 2D Helmholtz equations in the cylinder and two 2D Helmholtz equations in the exterior domain where the fields are coupled on the boundary. We prove uniqueness and existence of this differential system by formulating an equivalent system of integral equations using the direct method. We transform this system into a Fredholm type system of boundary integral equations in a Sobolev space setting. To handle the hypersingular operators we take advantage of Maue's formula. Applying a collocation method we derive an efficient numerical scheme and provide accurate numerical results using as test cases transmission problems corresponding to analytic fields derived from fundamental solutions.
\end{abstract}

1. Introduction. An interesting area of electromagnetism for its applications and the arising theoretical problems is the scattering process from obliquely incident time-harmonic plane waves by an infinitely long cylinder. The basic waves in the propagation domain satisfy Maxwell's equations $[\mathbf{1}, \mathbf{3}, \mathbf{1 6}, \mathbf{1 8}]$ and, due to the symmetry of

2010 AMS Mathematics subject classification. Primary 35P25, 35Q61, 45B05, 45F15, 78A25.

Keywords and phrases. Direct electromagnetic scattering, oblique incidence, integral equation method, hypersingular operator.

The research of the first author was supported by the program of NTUA for sabbatical visits.

Received by the editors on September 14, 2015. 
the problem, it is equivalent to finding two scalar fields satisfying a pair of two-dimensional Helmholtz equations with different wavenumbers. The complication appears in the boundary conditions. Even for the case of a perfect conductor, tangential derivatives appear in the boundary conditions which make the analysis more difficult. There are many studies providing analytical or numerical solutions $[\mathbf{2}, \mathbf{1 3}$, $19,20,21,22,24,25]$. The proposed methods are based on specific geometries or well known numerical schemes without examining the well-posedness of the corresponding boundary value problem.

Recently, Wang and Nakamura [23] used a more elegant theoretical analysis to prove well-posedness of the problem based on the integral equation approach. They proved theoretical and numerical results for the case of the homogeneous impedance cylinder using integral equations. For the theoretical analysis they used properties of the Cauchy singular integrals and proved that the system derived is of Fredholm type with index zero. For the numerical results they applied a specific decomposition of the kernels and formulations using Hilbert's and Symm's integral operators. Considering trigonometric interpolation, they introduced an efficient numerical scheme.

The case for general dielectric cylinders is not considered yet; however, the same authors, in a later work [17], investigated a more complicated model also having a non-homogeneous part, in the sense that the permittivity and the permeability of the exterior medium are non-constants and smooth in a bounded domain surrounding the cylinder. The main theoretical analysis providing uniqueness and existence in non-homogeneous materials is much harder. For the wellposedness they used the Lax-Phillips method [7].

In this work, we examine the case of the infinite dielectric cylinder illuminated by a transverse magnetic polarized electromagnetic plane wave, known as oblique incidence. More precisely, in the second section starting from Maxwell's equations we initially describe the derivation of the mathematical model for the scattering process from obliquely incident time-harmonic plane waves for the case of infinite inhomogeneous cylinder. We assume that transmission conditions hold on the boundary. The boundary conditions involve normal and tangential derivatives of the fields. 
In Section 3, we formulate the direct problem in differential form. We derive the Helmholtz equations and the exact form of the boundary conditions in the case of homogeneous cylinder. We prove that the problem is uniquely solvable using Green's formulas and Rellich's lemma. Considering the direct method, initially applied in transmission problems in $[\mathbf{5}, \mathbf{8}, \mathbf{9}]$, we formulate the problem into an equivalent system of integral equations. We show that this system is of Fredholm type in an appropriate Sobolev space setting. Due to uniqueness of the boundary value problem, existence follows from the Fredholm alternative. The system consists of compact, singular and hypersingular operators. We consider Maue's formula [14], as in the case of the normal derivative of the double layer potential, to reduce the hypersingularity of the tangential derivative of the double layer potential.

In the last section we investigate numerically the problem by a collocation method based on Kress's method for the two-dimensional integral equation with strongly singular operators [10]. We transform the system of integral equations to a linear system by parametrizing the operators and considering well-known quadrature rules. We derive accurate numerical results for the four fields, interior and exterior, and we numerically compute the far-field patterns of the two exterior fields computed for a specific boundary value problem. Namely, we consider boundary data corresponding to analytic fields derived from point sources, where the interior and exterior fields have singularities outside of their domain of consideration.

2. Formulation of the direct scattering problem for an inhomogeneous cylinder. We consider the scattering problem of an electromagnetic wave by a penetrable cylinder in $\mathbb{R}^{3}$. Let $\mathbf{x}=$ $(x, y, z) \in \mathbb{R}^{3}$. Then we model the cylinder as $\Omega_{\text {int }}=\{\mathbf{x}:(x, y) \in$ $\Omega, z \in \mathbb{R}\} 0$, where $\Omega$ is a bounded domain in $\mathbb{R}^{2}$ with smooth boundary $\Gamma$. The cylinder $\Omega_{\text {int }}$ is oriented parallel to the $z$-axis and $\Omega$ is its horizontal cross section. We assume constant permittivity $\epsilon_{0}$ and permeability $\mu_{0}$ for the exterior domain $\Omega_{\text {ext }}:=\mathbb{R}^{3} \backslash \bar{\Omega}_{\text {int }}$. The interior domain $\Omega_{\text {int }}$ is characterized by the electric constants $\mu(\mathbf{x})=\mu(x, y)$ and $\epsilon(\mathbf{x})=\epsilon(x, y)$ for all $(x, y) \in \Omega, z \in \mathbb{R}$.

We define for $\mathbf{x} \in \Omega_{\text {ext }}, t \in \mathbb{R}$, the magnetic field $\mathbf{H}^{\text {ext }}(\mathbf{x}, t)$ and electric field $\mathbf{E}^{\mathrm{ext}}(\mathbf{x}, t)$ and, equivalently, the interior fields $\mathbf{H}^{\mathrm{int}}(\mathbf{x}, t)$ 
and $\mathbf{E}^{\text {int }}(\mathbf{x}, t)$ for $\mathbf{x} \in \Omega_{\text {int }}, t \in \mathbb{R}$. Then, these fields satisfy the Maxwell's equations

$$
\begin{array}{cc}
\nabla \times \mathbf{E}^{\mathrm{ext}}+\mu_{0} \frac{\partial \mathbf{H}^{\mathrm{ext}}}{\partial t}=0, & \mathbf{x} \in \Omega_{\mathrm{ext}}, \\
\nabla \times \mathbf{H}^{\mathrm{ext}}-\epsilon_{0} \frac{\partial \mathbf{E}^{\mathrm{ext}}}{\partial t}=0, & \mathbf{x} \in \Omega_{\mathrm{ext}}, \\
\nabla \times \mathbf{E}^{\mathrm{int}}+\mu \frac{\partial \mathbf{H}^{\mathrm{int}}}{\partial t}=0, & \mathbf{x} \in \Omega_{\mathrm{int}}, \\
\nabla \times \mathbf{H}^{\text {int }}-\epsilon \frac{\partial \mathbf{E}^{\text {int }}}{\partial t}=0, & \mathbf{x} \in \Omega_{\text {int }} .
\end{array}
$$

On the boundary $\Gamma$, we consider transmission conditions

$$
\widehat{\boldsymbol{n}} \times \mathbf{E}^{\mathrm{int}}=\widehat{\boldsymbol{n}} \times \mathbf{E}^{\mathrm{ext}}, \quad \widehat{\boldsymbol{n}} \times \mathbf{H}^{\mathrm{int}}=\widehat{\boldsymbol{n}} \times \mathbf{H}^{\mathrm{ext}}, \quad \mathrm{x} \in \Gamma,
$$

where $\widehat{\boldsymbol{n}}$ is the outward normal vector, directed into $\Omega_{\text {ext }}$.

In order to take advantage of the symmetry of the specific medium, we probe the cylinder with an incident transverse magnetic (TM) polarized electromagnetic plane wave, the so-called oblique incidence in the literature. An arbitrary time-harmonic incident electromagnetic plane wave has the form:

$$
\begin{aligned}
& \mathbf{E}^{\mathrm{inc}}(\mathbf{x}, t ; \widehat{\boldsymbol{d}}, \widehat{\boldsymbol{p}})=\frac{1}{k_{0}^{2} \sqrt{\epsilon_{0}}} \nabla \times \nabla \times\left(\widehat{\boldsymbol{p}} e^{i k_{0} \mathbf{x} \cdot \widehat{\boldsymbol{d}}}\right) e^{-i \omega t}, \\
& \mathbf{H}^{\mathrm{inc}}(\mathbf{x}, t ; \widehat{\boldsymbol{d}}, \widehat{\boldsymbol{p}})=\frac{1}{i k_{0} \sqrt{\mu_{0}}} \nabla \times\left(\widehat{\boldsymbol{p}} e^{i k_{0} \mathbf{x} \cdot \widehat{\boldsymbol{d}}}\right) e^{-i \omega t},
\end{aligned}
$$

where $\omega>0$ is the frequency, $k_{0}=\omega \sqrt{\mu_{0} \epsilon_{0}}$ is the wave number in the exterior domain, $\widehat{\boldsymbol{p}}$ is the polarization vector and $\widehat{\boldsymbol{d}}$ the vector describing the incident direction, satisfying $\widehat{\boldsymbol{d}} \perp \widehat{\boldsymbol{p}}$.

In the following, due to the linearity of the problem, we suppress the time-dependence and we consider the fields only as functions of the space variable $\mathbf{x}$. In order to describe the incident fields for the specific TM polarization, we define by $\theta$ the incident angle with respect to the negative $z$ axis and by $\phi$ the polar angle of $\widehat{d}$ (in spherical coordinates). Then $\widehat{\boldsymbol{d}}=(\sin \theta \cos \phi, \sin \theta \sin \phi,-\cos \theta)$ and $\widehat{\boldsymbol{p}}=(\cos \theta \cos \phi, \cos \theta \sin \phi, \sin \theta)$, assuming that $\theta \in(0, \pi / 2) \cup(\pi / 2, \pi)$. 
Hence, we obtain

$$
\begin{aligned}
& \mathbf{E}^{\text {inc }}(\mathbf{x} ; \widehat{\boldsymbol{d}}, \widehat{\boldsymbol{p}})=\frac{1}{\sqrt{\epsilon_{0}}} \widehat{\boldsymbol{d}} \times \widehat{\boldsymbol{p}} \times \widehat{\boldsymbol{d}} e^{i k_{0} \mathbf{x} \cdot \widehat{\boldsymbol{d}}}=\frac{1}{\sqrt{\epsilon_{0}}} \widehat{\boldsymbol{p}} e^{i k_{0} \mathbf{x} \cdot \widehat{\boldsymbol{d}}} \\
& \mathbf{H}^{\mathrm{inc}}(\mathbf{x} ; \widehat{\boldsymbol{d}}, \widehat{\boldsymbol{p}})=\frac{1}{\sqrt{\mu_{0}}} \widehat{\boldsymbol{d}} \times \widehat{\boldsymbol{p}} e^{i k_{0} \mathbf{x} \cdot \widehat{\boldsymbol{d}}}=\frac{1}{\sqrt{\mu_{0}}}(\sin \phi,-\cos \phi, 0) e^{i k_{0} \mathbf{x} \cdot \widehat{\boldsymbol{d}}} .
\end{aligned}
$$

Taking into account the cylindrical symmetry of the medium and the $z$-independence of the electric coefficients, we express the incident fields as separable functions of $(x, y)$ and $z$. Thus, we define $\beta=$ $k_{0} \cos \theta$ and $\kappa_{0}=\sqrt{k_{0}^{2}-\beta^{2}}=k_{0} \sin \theta$, and it follows that the incident fields can be decomposed to

$$
\begin{aligned}
\mathbf{E}^{\text {inc }}(\mathbf{x} ; \widehat{\boldsymbol{d}}, \widehat{\boldsymbol{p}}) & =\mathbf{e}^{\mathrm{inc}}(x, y) e^{-i \beta z}, \\
\mathbf{H}^{\mathrm{inc}}(\mathbf{x} ; \widehat{\boldsymbol{d}}, \widehat{\boldsymbol{p}}) & =\mathbf{h}^{\mathrm{inc}}(x, y) e^{-i \beta z}
\end{aligned}
$$

where

$$
\begin{aligned}
\mathbf{e}^{\text {inc }}(x, y) & =\frac{1}{\sqrt{\epsilon_{0}}} \widehat{\boldsymbol{p}} e^{i \kappa_{0}(x \cos \phi+y \sin \phi)} \\
\mathbf{h}^{\text {inc }}(x, y) & =\frac{1}{\sqrt{\mu_{0}}}(\sin \phi,-\cos \phi, 0) e^{i \kappa_{0}(x \cos \phi+y \sin \phi)}
\end{aligned}
$$

Now, we are in a position to transform equations (2.1) into a system of equations only for the z-component of the electric and magnetic fields. Firstly, we see that for the specific illumination of the form (2.2), using separation of variables, the scattered fields also take the form:

$$
\begin{array}{ll}
\mathbf{E}^{\mathrm{sc}}(\mathbf{x} ; \widehat{\boldsymbol{d}}, \widehat{\boldsymbol{p}})=\mathbf{e}^{\mathrm{sc}}(x, y) e^{-i \beta z}, & \mathbf{x} \in \Omega_{\mathrm{ext}}, \\
\mathbf{H}^{\mathrm{sc}}(\mathbf{x} ; \widehat{\boldsymbol{d}}, \widehat{\boldsymbol{p}})=\mathbf{h}^{\mathrm{sc}}(x, y) e^{-i \beta z}, & \mathbf{x} \in \Omega_{\mathrm{ext}},
\end{array}
$$

where $\mathbf{e}^{\mathrm{sc}}=\left(e_{1}^{s c}, e_{2}^{s c}, e_{3}^{s c}\right)$ and $\mathbf{h}^{\mathrm{sc}}=\left(h_{1}^{s c}, h_{2}^{s c}, h_{3}^{s c}\right)$. Then, the exterior fields are given by

$$
\begin{array}{rlrl}
\mathbf{E}^{\mathrm{ext}}(\mathbf{x} ; \widehat{\boldsymbol{d}}, \widehat{\boldsymbol{p}}) & =\left(\mathbf{e}^{\mathrm{sc}}(x, y)+\mathbf{e}^{\mathrm{inc}}(x, y)\right) e^{-i \beta z} & & \\
& =\mathbf{e}^{\mathrm{ext}}(x, y) e^{-i \beta z}, & \mathbf{x} \in \Omega_{\mathrm{ext}}, \\
\mathbf{H}^{\operatorname{ext}}(\mathbf{x} ; \widehat{\boldsymbol{d}}, \widehat{\boldsymbol{p}}) & =\left(\mathbf{h}^{\mathrm{sc}}(x, y)+\mathbf{h}^{\mathrm{inc}}(x, y)\right) e^{-i \beta z} & & \\
& =\mathbf{e}^{\operatorname{ext}}(x, y) e^{-i \beta z}, & \mathbf{x} \in \Omega_{\mathrm{ext}} .
\end{array}
$$


Equivalently, the interior fields are represented by

$$
\begin{aligned}
\mathbf{E}^{\mathrm{int}}(\mathbf{x} ; \widehat{\boldsymbol{d}}, \widehat{\boldsymbol{p}})=\mathbf{e}^{\mathrm{int}}(x, y) e^{-i \beta z}, & \mathbf{x} \in \Omega_{\mathrm{int}}, \\
\mathbf{H}^{\mathrm{int}}(\mathbf{x} ; \widehat{\boldsymbol{d}}, \widehat{\boldsymbol{p}})=\mathbf{h}^{\mathrm{int}}(x, y) e^{-i \beta z}, & \mathbf{x} \in \Omega_{\mathrm{int}},
\end{aligned}
$$

where $\mathbf{e}^{\mathrm{int}}=\left(e_{1}^{\mathrm{int}}, e_{2}^{\mathrm{int}}, e_{3}^{\mathrm{int}}\right)$ and $\mathbf{h}^{\mathrm{int}}=\left(h_{1}^{\mathrm{int}}, h_{2}^{\mathrm{int}}, h_{3}^{\mathrm{int}}\right)$.

For any field of the form

$$
\begin{aligned}
& \mathbf{E}(\mathbf{x} ; \widehat{\boldsymbol{d}}, \widehat{\boldsymbol{p}})=\mathbf{e}(x, y) e^{-i \beta z}, \quad \mathbf{x} \in \mathbb{R}^{3}, \\
& \mathbf{H}(\mathbf{x} ; \widehat{\boldsymbol{d}}, \widehat{\boldsymbol{p}})=\mathbf{h}(x, y) e^{-i \beta z}, \quad \mathbf{x} \in \mathbb{R}^{3},
\end{aligned}
$$

we consider the Maxwell's equations in $\mathbb{R}^{3}$ for arbitrary $\epsilon, \mu$ and $k^{2}=\mu \epsilon \omega^{2}-\beta^{2}$ (we remark here the space dependence of $\epsilon, \mu$ ). Then, following [17], we obtain the relations

$$
\begin{aligned}
& e_{1}(x, y)=-\frac{1}{k^{2}}\left(i \beta \frac{\partial e_{3}}{\partial x}(x, y)-i \mu \omega \frac{\partial h_{3}}{\partial y}(x, y)\right), \\
& e_{2}(x, y)=-\frac{1}{k^{2}}\left(i \beta \frac{\partial e_{3}}{\partial y}(x, y)+i \mu \omega \frac{\partial h_{3}}{\partial x}(x, y)\right), \\
& h_{1}(x, y)=-\frac{1}{k^{2}}\left(i \beta \frac{\partial h_{3}}{\partial x}(x, y)+i \epsilon \omega \frac{\partial e_{3}}{\partial y}(x, y)\right), \\
& h_{2}(x, y)=-\frac{1}{k^{2}}\left(i \beta \frac{\partial h_{3}}{\partial y}(x, y)-i \epsilon \omega \frac{\partial e_{3}}{\partial x}(x, y)\right) .
\end{aligned}
$$

Substituting (2.3) in (2.1), we have that the pair $\left(e_{3}, h_{3}\right)$ satisfies the equations

$$
\begin{gathered}
\frac{k^{2}}{\epsilon \omega} \nabla \cdot\left(\frac{\epsilon \omega}{k^{2}} \nabla e_{3}\right)+\frac{k^{2}}{\epsilon \omega} J \nabla\left(\frac{\beta}{k^{2}}\right) \cdot \nabla h_{3}+k^{2} e_{3}=0, \\
\frac{k^{2}}{\mu \omega} \nabla \cdot\left(\frac{\mu \omega}{k^{2}} \nabla h_{3}\right)-\frac{k^{2}}{\mu \omega} J \nabla\left(\frac{\beta}{k^{2}}\right) \cdot \nabla e_{3}+k^{2} h_{3}=0,
\end{gathered}
$$

where

$$
\mathbf{J}=\left(\begin{array}{rr}
0 & 1 \\
-1 & 0
\end{array}\right)
$$

The interior and exterior domains are characterized by different 
wavenumbers, given by

$$
k^{2}(\mathbf{x})= \begin{cases}k_{\text {int }}^{2}(\mathbf{x}):=\mu(x, y) \epsilon(x, y) \omega^{2}-\beta^{2}, & \mathbf{x} \in \Omega_{\text {int }}, \\ k_{\text {ext }}^{2}(\mathbf{x}):=\mu_{0} \epsilon_{0} \omega^{2}-\beta^{2}=\kappa_{0}^{2}, & \mathbf{x} \in \Omega_{\text {ext }}\end{cases}
$$

In this section, for completeness in the formulation of the direct problem, we keep the space dependence of $k_{\text {int }}$. Later, we consider only the case of constant parameters. Here, we have to assume that $\mu(\mathbf{x}) \epsilon(\mathbf{x})>\epsilon_{0} \mu_{0} \cos \theta$ in order to have $\inf _{\mathbf{x}} k_{\text {int }}^{2}(\mathbf{x})>0$. Thus, the fields $e_{3}^{\text {ext }}(x, y)$ and $h_{3}^{\text {ext }}(x, y)$ satisfy

$$
\Delta e_{3}^{\text {ext }}+\kappa_{0}^{2} e_{3}^{\text {ext }}=0, \quad \Delta h_{3}^{\text {ext }}+\kappa_{0}^{2} h_{3}^{\text {ext }}=0, \quad \mathbf{x} \in \Omega_{\text {ext }},
$$

and the interior fields

$$
\begin{aligned}
\frac{k_{\text {int }}^{2}(\mathbf{x})}{\epsilon(\mathbf{x})} \nabla \cdot\left(\frac{\epsilon(\mathbf{x})}{k_{\text {int }}^{2}(\mathbf{x})} \nabla e_{3}^{\text {int }}\right) \\
+\frac{k_{\text {int }}^{2}(\mathbf{x})}{\epsilon(\mathbf{x}) \omega} \mathbf{J} \nabla\left(\frac{\beta}{k_{\text {int }}^{2}(\mathbf{x})}\right) \cdot \nabla h_{3}^{\text {int }}+k_{\text {int }}^{2}(\mathbf{x}) e_{3}^{\text {int }}=0, \quad \mathbf{x} \in \Omega_{\text {int }}, \\
\frac{k_{\text {int }}^{2}(\mathbf{x})}{\mu(\mathbf{x})} \nabla \cdot\left(\frac{\mu(\mathbf{x})}{k_{\text {int }}^{2}(\mathbf{x})} \nabla h_{3}^{\text {int }}\right) \\
-\frac{k_{\text {int }}^{2}(\mathbf{x})}{\mu(\mathbf{x}) \omega} \mathbf{J} \nabla\left(\frac{\beta}{k_{\text {int }}^{2}(\mathbf{x})}\right) \cdot \nabla e_{3}^{\text {int }}+k_{\text {int }}^{2}(\mathbf{x}) h_{3}^{\text {int }}=0, \quad \mathbf{x} \in \Omega_{\text {int }} .
\end{aligned}
$$

Now, we are going to derive the exact form of the boundary conditions. We introduce the notation: $\mathbf{e}_{t}=\widehat{\boldsymbol{x}} e_{1}+\widehat{\boldsymbol{y}} e_{2}, \mathbf{h}_{t}=$ $\widehat{\boldsymbol{x}} h_{1}+\widehat{\boldsymbol{y}} h_{2}$ and $\nabla_{t}=\widehat{\boldsymbol{x}}(\partial / \partial x)+\widehat{\boldsymbol{y}}(\partial / \partial y)$, where $\widehat{\boldsymbol{x}}, \widehat{\boldsymbol{y}}$ denote the unit vectors in $\mathbb{R}^{2}$. Let $(\widehat{\boldsymbol{n}}, \widehat{\boldsymbol{\tau}}, \widehat{\boldsymbol{z}})$ be a local coordinate system, where $\widehat{\boldsymbol{n}}=\left(n_{1}, n_{2}\right)$ is the outward normal vector and $\widehat{\boldsymbol{\tau}}=\left(-n_{2}, n_{1}\right)$ the outward tangent vector on $\Gamma$. Then, from (2.3), we obtain

$$
\begin{aligned}
& \widehat{\boldsymbol{\tau}} \cdot \mathbf{e}_{t}=-\frac{1}{k^{2}}\left(i \mu \omega \widehat{\boldsymbol{n}} \cdot \nabla_{t} h_{3}+i \beta \widehat{\boldsymbol{\tau}} \cdot \nabla_{t} e_{3}\right), \\
& \widehat{\boldsymbol{\tau}} \cdot \mathbf{h}_{t}=-\frac{1}{k^{2}}\left(-i \epsilon \omega \widehat{\boldsymbol{n}} \cdot \nabla_{t} e_{3}+i \beta \widehat{\boldsymbol{\tau}} \cdot \nabla_{t} h_{3}\right),
\end{aligned}
$$

using that $\widehat{\boldsymbol{\tau}} \cdot\left(\widehat{\boldsymbol{z}} \times \nabla_{t}\right)=\widehat{\boldsymbol{n}} \cdot \nabla_{t} \cdot$. 
We observe, setting zero to the $z$-component of $\widehat{\boldsymbol{n}}, \widehat{\boldsymbol{\tau}}$ in $\mathbb{R}^{3}$, that

$$
\begin{aligned}
& \widehat{\boldsymbol{n}} \times \mathbf{E}=-e_{3} \widehat{\boldsymbol{\tau}}+\left(n_{1} e_{2}-n_{2} e_{1}\right) \widehat{\boldsymbol{z}}, \\
& \widehat{\boldsymbol{n}} \times \mathbf{H}=-h_{3} \widehat{\boldsymbol{\tau}}+\left(n_{1} h_{2}-n_{2} h_{1}\right) \widehat{\boldsymbol{z}} .
\end{aligned}
$$

Then, from (2.3) and (2.6), we derive

$$
\begin{aligned}
\widehat{\boldsymbol{n}} \times \mathbf{E}^{\mathrm{ext}} & =-e_{3}^{\operatorname{ext} \widehat{\boldsymbol{\tau}}}+\widehat{\boldsymbol{\tau}} \cdot \mathbf{e}_{t}^{\operatorname{ext}} \widehat{\boldsymbol{z}}, \\
\widehat{\boldsymbol{n}} \times \mathbf{H}^{\mathrm{ext}} & =-h_{3}^{\operatorname{ext}} \widehat{\boldsymbol{\tau}}+\widehat{\boldsymbol{\tau}} \cdot \mathbf{h}_{t}^{\operatorname{ext}} \widehat{\boldsymbol{z}},
\end{aligned}
$$

for the exterior fields, where $\mathbf{e}_{t}^{\text {ext }}:=\widehat{\boldsymbol{x}} e_{1}^{\text {ext }}+\widehat{\boldsymbol{y}} e_{2}^{\text {ext }}, \mathbf{h}_{t}^{\text {ext }}:=\widehat{\boldsymbol{x}} h_{1}^{\text {ext }}+$ $\widehat{\boldsymbol{y}} h_{2}^{\mathrm{ext}}$ and

$$
\begin{aligned}
& \widehat{\boldsymbol{n}} \times \mathbf{E}^{\mathrm{int}}=-e_{3}^{\mathrm{int}} \widehat{\boldsymbol{\tau}}+\widehat{\boldsymbol{\tau}} \cdot \mathbf{e}_{t}^{\mathrm{int}} \widehat{\boldsymbol{z}}, \\
& \widehat{\boldsymbol{n}} \times \mathbf{H}^{\mathrm{int}}=-h_{3}^{\mathrm{int}} \widehat{\boldsymbol{\tau}}+\widehat{\boldsymbol{\tau}} \cdot \mathbf{h}_{t}^{\mathrm{int}} \widehat{\boldsymbol{z}} .
\end{aligned}
$$

for the interior fields, where $\mathbf{e}_{t}^{\mathrm{int}}:=\widehat{\boldsymbol{x}} e_{1}^{\mathrm{int}}+\widehat{\boldsymbol{y}} e_{2}^{\mathrm{int}}, \mathbf{h}_{t}^{\mathrm{int}}:=\widehat{\boldsymbol{x}} h_{1}^{\mathrm{int}}+\widehat{\boldsymbol{y}} h_{2}^{\mathrm{int}}$.

Here, we observe that the tangential forms of the fields can be written in terms of $\widehat{\boldsymbol{\tau}}$ and $\widehat{\boldsymbol{z}}$, two linear independent vectors. Thus, the boundary condition

$$
\widehat{\boldsymbol{n}} \times \mathbf{E}^{\mathrm{int}}=\widehat{\boldsymbol{n}} \times \mathbf{E}^{\mathrm{ext}}, \quad \mathrm{x} \in \Gamma,
$$

is equivalent to the system

$$
e_{3}^{\text {int }}=e_{3}^{\text {ext }}, \quad \widehat{\boldsymbol{\tau}} \cdot \mathbf{e}_{t}^{\text {int }}=\widehat{\boldsymbol{\tau}} \cdot \mathbf{e}_{t}^{\text {ext }}, \quad \mathbf{x} \in \Gamma,
$$

and equivalently for the magnetic fields

$$
h_{3}^{\text {int }}=h_{3}^{\text {ext }}, \quad \widehat{\boldsymbol{\tau}} \cdot \mathbf{h}_{t}^{\text {int }}=\widehat{\boldsymbol{\tau}} \cdot \mathbf{h}_{t}^{\text {ext }}, \quad \mathbf{x} \in \Gamma .
$$

We define

$$
\frac{\partial}{\partial n}=\widehat{\boldsymbol{n}} \cdot \nabla_{t}, \quad \frac{\partial}{\partial \tau}=\widehat{\boldsymbol{\tau}} \cdot \nabla_{t},
$$

and we rewrite the above boundary conditions as

$$
\frac{\mu(\mathbf{x})}{k_{\text {int }}^{2}(\mathbf{x})} \omega \frac{\partial h_{3}^{\mathrm{int}}}{\partial n}+\frac{\beta}{k_{\text {int }}^{2}(\mathbf{x})} \frac{\partial e_{3}^{\mathrm{int}}}{\partial \tau}=\frac{\mu_{0}}{\kappa_{0}^{2}} \omega \frac{\partial h_{3}^{\mathrm{ext}}}{\partial n}+\frac{\beta}{\kappa_{0}^{2}} \frac{\partial e_{3}^{\mathrm{ext}}}{\partial \tau}, \quad \mathbf{x} \in \Gamma,
$$


and

$$
\begin{aligned}
h_{3}^{\text {int }}=h_{3}^{\text {ext }}, & \mathbf{x} \in \Gamma, \\
\frac{\epsilon(\mathbf{x})}{k_{\text {int }}^{2}(\mathbf{x})} \omega \frac{\partial e_{3}^{\text {int }}}{\partial n}-\frac{\beta}{k_{\text {int }}^{2}(\mathbf{x})} \frac{\partial h_{3}^{\text {int }}}{\partial \tau}=\frac{\epsilon_{0}}{\kappa_{0}^{2}} \omega \frac{\partial e_{3}^{\text {ext }}}{\partial n}-\frac{\beta}{\kappa_{0}^{2}} \frac{\partial h_{3}^{\text {ext }}}{\partial \tau}, & \mathbf{x} \in \Gamma .
\end{aligned}
$$

To ensure that the scattered fields are outgoing, the components must satisfy in addition the radiation conditions in $\mathbb{R}^{2}$ :

$$
\begin{aligned}
& \lim _{r \rightarrow \infty} \sqrt{r}\left(\frac{\partial e_{3}^{\mathrm{sc}}}{\partial r}-i \kappa_{0} e_{3}^{\mathrm{sc}}\right)=0, \\
& \lim _{r \rightarrow \infty} \sqrt{r}\left(\frac{\partial h_{3}^{\mathrm{sc}}}{\partial r}-i \kappa_{0} h_{3}^{\mathrm{sc}}\right)=0,
\end{aligned}
$$

where $r=|(x, y)|$ uniformly over all directions.

Thus, the direct transmission problem for oblique incident wave, is to find the fields $h_{3}^{\mathrm{int}}, h_{3}^{\mathrm{sc}}, e_{3}^{\mathrm{int}}$ and $e_{3}^{\mathrm{sc}}$ which satisfy equations $(2.4)$ and (2.5), the transmission conditions (2.7) and (2.8) and the radiation conditions $(2.9)$.

We remark here that, since we consider TM polarized wave, see equation (2.2), the incident fields for $\mathbf{x} \in \Omega_{\text {ext }}$ are simplified to

$$
e_{3}^{\mathrm{inc}}(x, y)=\frac{1}{\sqrt{\epsilon_{0}}} \sin \theta e^{i \kappa_{0}(x \cos \phi+y \sin \phi)}, \quad h_{3}^{\mathrm{inc}}(x, y)=0 .
$$

3. The direct problem for a homogeneous cylinder using the integral equation method. From now on, $\mathbf{x} \in \mathbb{R}^{2}$. In this section, we consider the simplified version where $\mu(\mathbf{x})=\mu_{1}$ and $\epsilon(\mathbf{x})=\epsilon_{1}$ are constant in the interior domain. To simplify the following analysis, we set $\Omega_{1}=\Omega \subset \mathbb{R}^{2}, \Omega_{0}=\mathbb{R}^{2} \backslash \Omega$ and

$$
\begin{array}{lll}
u_{0}(\mathbf{x})=e_{3}^{\mathrm{sc}}(\mathbf{x}), & v_{0}(\mathbf{x})=h_{3}^{\mathrm{sc}}(\mathbf{x}), & \mathbf{x} \in \Omega_{0}, \\
u_{1}(\mathbf{x})=e_{3}^{\text {int }}(\mathbf{x}), & v_{1}(\mathbf{x})=h_{3}^{\text {int }}(\mathbf{x}), & \mathbf{x} \in \Omega_{1} .
\end{array}
$$

In the following, $j=0,1$ counts for the exterior $\left(\mathbf{x} \in \Omega_{0}\right)$ and interior domains $\left(x \in \Omega_{1}\right)$, respectively. Then, the direct scattering problem, presented in the previous section, is modified to

$$
\Delta u_{j}+\kappa_{j}^{2} u_{j}=0, \quad \Delta v_{j}+\kappa_{j}^{2} v_{j}=0, \quad \mathbf{x} \in \Omega_{j},
$$


for $j=0,1$ where $\kappa_{1}^{2}=\mu_{1} \epsilon_{1} \omega^{2}-\beta^{2}$, with boundary conditions

$$
\begin{aligned}
u_{1} & =u_{0}+e_{3}^{\text {inc }}, & & \mathbf{x} \in \Gamma \\
\widetilde{\mu}_{1} \omega \frac{\partial v_{1}}{\partial n}+\beta_{1} \frac{\partial u_{1}}{\partial \tau} & =\widetilde{\mu}_{0} \omega \frac{\partial v_{0}}{\partial n}+\beta_{0} \frac{\partial u_{0}}{\partial \tau}+\beta_{0} \frac{\partial e_{3}^{\text {inc }}}{\partial \tau}, & & \mathbf{x} \in \Gamma, \\
v_{1} & =v_{0}, & & \mathbf{x} \in \Gamma, \\
\widetilde{\epsilon}_{1} \omega \frac{\partial u_{1}}{\partial n}-\beta_{1} \frac{\partial v_{1}}{\partial \tau} & =\widetilde{\epsilon}_{0} \omega \frac{\partial u_{0}}{\partial n}+\widetilde{\epsilon}_{0} \omega \frac{\partial e_{3}^{\text {inc }}}{\partial n}-\beta_{0} \frac{\partial v_{0}}{\partial \tau}, & & \mathbf{x} \in \Gamma,
\end{aligned}
$$

where $\widetilde{\mu}_{j}=\mu_{j} / \kappa_{j}^{2}, \widetilde{\epsilon}_{j}=\epsilon_{j} / \kappa_{j}^{2}, \beta_{j}=\beta / \kappa_{j}^{2}$, and the radiation conditions

$$
\begin{aligned}
& \lim _{r \rightarrow \infty} \sqrt{r}\left(\frac{\partial u_{0}}{\partial r}-i \kappa_{0} u_{0}\right)=0 \\
& \lim _{r \rightarrow \infty} \sqrt{r}\left(\frac{\partial v_{0}}{\partial r}-i \kappa_{0} v_{0}\right)=0 .
\end{aligned}
$$

Theorem 3.1. If $\kappa_{1}^{2}$ is not an interior Dirichlet eigenvalue, then the problem (3.1)-(3.3) has at most one solution.

Proof. It is sufficient to show that, if $u_{0}, v_{0}, u_{1}, v_{1}$ solve the homogeneous problem (3.1)-(3.3), that is, for $e_{3}^{\text {inc }}=0$, then $u_{0}=$ $v_{0}=0$ in $\Omega_{0}$ and $u_{1}=v_{1}=0$ in $\Omega_{1}$. Let $S_{r}$ be a disk with radius $r$, boundary $\Gamma_{r}$, centered at the origin and containing $\Omega_{1}$. We set $\Omega_{r}=S_{r} \backslash \bar{\Omega}_{1}$, see Figure 1 .

The boundary conditions of the homogeneous problem read

$$
\begin{aligned}
u_{1} & =u_{0}, & & \mathbf{x} \in \Gamma, \\
\widetilde{\mu}_{1} \frac{\partial v_{1}}{\partial n}-\widetilde{\mu}_{0} \frac{\partial v_{0}}{\partial n} & =-\frac{\beta_{1}}{\omega} \frac{\partial u_{1}}{\partial \tau}+\frac{\beta_{0}}{\omega} \frac{\partial u_{0}}{\partial \tau}, & & \mathbf{x} \in \Gamma, \\
v_{1} & =v_{0}, & \mathbf{x} & \in \Gamma, \\
\widetilde{\epsilon}_{1} \frac{\partial u_{1}}{\partial n}-\widetilde{\epsilon}_{0} \frac{\partial u_{0}}{\partial n} & =\frac{\beta_{1}}{\omega} \frac{\partial v_{1}}{\partial \tau}-\frac{\beta_{0}}{\omega} \frac{\partial v_{0}}{\partial \tau}, & & \mathbf{x} \in \Gamma .
\end{aligned}
$$




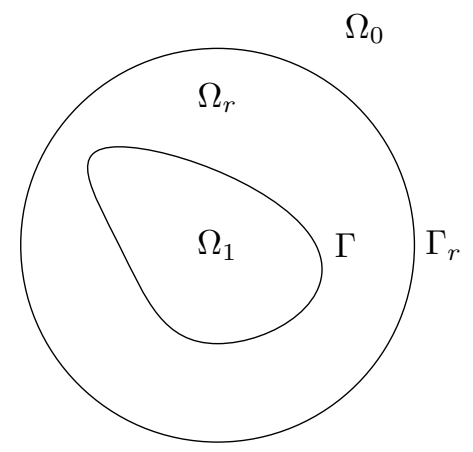

Figure 1 . The set $\Omega_{r}$.

We apply Green's first identity in $\Omega_{1}$ and considering (3.1) we obtain

$$
\begin{aligned}
\widetilde{\epsilon}_{1} \int_{\Gamma} u_{1} \frac{\partial \bar{u}_{1}}{\partial n} d s & =\widetilde{\epsilon}_{1} \int_{\Omega_{1}}\left(\left|\nabla u_{1}\right|^{2}+u_{1} \Delta \bar{u}_{1}\right) d \mathbf{x}, \\
& =\widetilde{\epsilon}_{1} \int_{\Omega_{1}}\left(\left|\nabla u_{1}\right|^{2}-\kappa_{1}^{2}\left|u_{1}\right|^{2}\right) d \mathbf{x}, \\
\widetilde{\mu}_{1} \int_{\Gamma} v_{1} \frac{\partial \bar{v}_{1}}{\partial n} d s & =\widetilde{\mu}_{1} \int_{\Omega_{1}}\left(\left|\nabla v_{1}\right|^{2}+v_{1} \Delta \bar{v}_{1}\right) d \mathbf{x} \\
& =\widetilde{\mu}_{1} \int_{\Omega_{1}}\left(\left|\nabla v_{1}\right|^{2}-\kappa_{1}^{2}\left|v_{1}\right|^{2}\right) d \mathbf{x} .
\end{aligned}
$$

Similarly, Green's first identity in $\Omega_{r}$, together with equations (3.4) and (3.5) gives

$$
\begin{aligned}
\widetilde{\epsilon}_{0} \int_{\Gamma_{r}} u_{0} \frac{\partial \bar{u}_{0}}{\partial n} d s= & \widetilde{\epsilon}_{0} \int_{\Omega_{r}}\left(\left|\nabla u_{0}\right|^{2}+u_{0} \Delta \bar{u}_{0}\right) d \mathbf{x} \\
& +\widetilde{\epsilon}_{0} \int_{\Gamma} u_{0} \frac{\partial \bar{u}_{0}}{\partial n} d s \\
= & \widetilde{\epsilon}_{0} \int_{\Omega_{r}}\left(\left|\nabla u_{0}\right|^{2}-\kappa_{0}^{2}\left|u_{0}\right|^{2}\right) d \mathbf{x} \\
& +\int_{\Gamma} u_{0}\left(\widetilde{\epsilon}_{1} \frac{\partial \bar{u}_{1}}{\partial n}-\frac{\beta_{1}}{\omega} \frac{\partial \bar{v}_{1}}{\partial \tau}+\frac{\beta_{0}}{\omega} \frac{\partial \bar{v}_{0}}{\partial \tau}\right) d s
\end{aligned}
$$




$$
\begin{aligned}
= & \widetilde{\epsilon}_{0} \int_{\Omega_{r}}\left(\left|\nabla u_{0}\right|^{2}-\kappa_{0}^{2}\left|u_{0}\right|^{2}\right) d \mathbf{x} \\
& +\widetilde{\epsilon}_{1} \int_{\Omega_{1}}\left(\left|\nabla u_{1}\right|^{2}-\kappa_{1}^{2}\left|u_{1}\right|^{2}\right) d \mathbf{x} \\
& -\frac{\beta_{1}}{\omega} \int_{\Gamma} u_{1} \frac{\partial \bar{v}_{1}}{\partial \tau} d s+\frac{\beta_{0}}{\omega} \int_{\Gamma} u_{0} \frac{\partial \bar{v}_{0}}{\partial \tau} d s
\end{aligned}
$$

and

$$
\begin{aligned}
\widetilde{\mu}_{0} \int_{\Gamma_{r}} v_{0} \frac{\partial \bar{v}_{0}}{\partial n} d s= & \widetilde{\mu}_{0} \int_{\Omega_{r}}\left(\left|\nabla v_{0}\right|^{2}+v_{0} \Delta \bar{v}_{0}\right) d \mathbf{x} \\
& +\widetilde{\mu}_{0} \int_{\Gamma} v_{0} \frac{\partial \bar{v}_{0}}{\partial n} d s \\
= & \widetilde{\mu}_{0} \int_{\Omega_{r}}\left(\left|\nabla v_{0}\right|^{2}-\kappa_{0}^{2}\left|v_{0}\right|^{2}\right) d \mathbf{x} \\
& +\int_{\Gamma} v_{0}\left(\widetilde{\mu}_{1} \frac{\partial \bar{v}_{1}}{\partial n}-\frac{\beta_{0}}{\omega} \frac{\partial \bar{u}_{0}}{\partial \tau}+\frac{\beta_{1}}{\omega} \frac{\partial \bar{u}_{1}}{\partial \tau}\right) d s \\
= & \widetilde{\mu}_{0} \int_{\Omega_{r}}\left(\left|\nabla v_{0}\right|^{2}-\kappa_{0}^{2}\left|v_{0}\right|^{2}\right) d \mathbf{x} \\
& +\widetilde{\mu}_{1} \int_{\Omega_{0}}\left(\left|\nabla v_{1}\right|^{2}-\kappa_{1}^{2}\left|v_{1}\right|^{2}\right) d \mathbf{x}-\frac{\beta_{0}}{\omega} \int_{\Gamma} v_{0} \frac{\partial \bar{u}_{0}}{\partial \tau} d s \\
& +\frac{\beta_{1}}{\omega} \int_{\Gamma} v_{1} \frac{\partial \bar{u}_{1}}{\partial \tau} d s .
\end{aligned}
$$

We add the above two equations and, noting that

$$
-\int_{\Gamma} u_{1} \frac{\partial \bar{v}_{1}}{\partial \tau} d s=\overline{\int_{\Gamma} v_{1} \frac{\partial \bar{u}_{1}}{\partial \tau} d s}, \quad \int_{\Gamma} u_{0} \frac{\partial \bar{v}_{0}}{\partial \tau} d s=-\overline{\int_{\Gamma} v_{0} \frac{\partial \bar{u}_{0}}{\partial \tau} d s}
$$

we obtain

$$
\Im\left(\widetilde{\epsilon}_{0} \int_{\Gamma_{r}} u_{0} \frac{\partial \bar{u}_{0}}{\partial n} d s+\widetilde{\mu}_{0} \int_{\Gamma_{r}} v_{0} \frac{\partial \bar{v}_{0}}{\partial n} d s\right)=0,
$$

or, equivalently, using the radiation conditions (see [23, equation $(2.12)])$

$$
\lim _{r \rightarrow \infty} \int_{\Gamma_{r}}\left(\epsilon_{0}\left|u_{0}\right|^{2}+\tilde{\epsilon}_{0}\left|\frac{\partial u_{0}}{\partial n}\right|^{2}+\mu_{0}\left|v_{0}\right|^{2}+\tilde{\mu}_{0}\left|\frac{\partial v_{0}}{\partial n}\right|^{2}\right) d s=0 .
$$


Thus,

$$
\lim _{r \rightarrow \infty} \int_{\Gamma_{r}}\left|u_{0}\right|^{2} d s=\lim _{r \rightarrow \infty} \int_{\Gamma_{r}}\left|v_{0}\right|^{2} d s=0,
$$

and by Rellich's lemma it follows that $u_{0}=v_{0}=0$ in $\Omega_{0}$. Hence, $u_{0}=v_{0}=0$ in $\Gamma$ and $u_{1}=v_{1}=0$ in $\Gamma$ from the boundary conditions. Then, $u_{1}=v_{1}=0$ in $\Omega_{1}$ follows from the unique solvability of the interior Dirichlet problem, given the assumption of the theorem.

We define the fundamental solution of the Helmholtz equation in $\mathbb{R}^{2}$ :

$$
\Phi_{j}(\mathbf{x}, \mathbf{y})=\frac{i}{4} H_{0}^{(1)}\left(\kappa_{j}|\mathbf{x}-\mathbf{y}|\right), \quad \mathbf{x}, \mathbf{y} \in \Omega_{j}, \mathbf{x} \neq \mathbf{y},
$$

where $H_{0}^{(1)}$ is the Hankel function of the first kind and zero order. For a continuous density $f$, we introduce the single- and double-layer potentials defined by

$$
\begin{array}{ll}
\left(S_{j} f\right)(\mathbf{x})=\int_{\Gamma} \Phi_{j}(\mathbf{x}, \mathbf{y}) f(\mathbf{y}) d s(\mathbf{y}), & \mathbf{x} \in \Omega_{j}, \\
\left(D_{j} f\right)(\mathbf{x})=\int_{\Gamma} \frac{\partial \Phi_{j}}{\partial n(\mathbf{y})}(\mathbf{x}, \mathbf{y}) f(\mathbf{y}) d s(\mathbf{y}), & \mathbf{x} \in \Omega_{j},
\end{array}
$$

and their derivatives, normal and tangential as $\mathbf{x} \rightarrow \Gamma$, using the standard jump relations, see for example $[\mathbf{3}, \mathbf{6}]$

$$
\begin{array}{rlrl}
\frac{\partial}{\partial n}\left(S_{j} f\right)(\mathbf{x}) & =\int_{\Gamma} \frac{\partial \Phi_{j}}{\partial n(\mathbf{x})}(\mathbf{x}, \mathbf{y}) f(\mathbf{y}) d s(\mathbf{y}) \mp \frac{1}{2} f(\mathbf{x}) & & \\
& :=\left(N S_{j} f\right)(\mathbf{x}) \mp \frac{1}{2} f(\mathbf{x}), & \mathbf{x} \in \Gamma, \\
\frac{\partial}{\partial n}\left(D_{j} f\right)(\mathbf{x}) & =\int_{\Gamma} \frac{\partial^{2} \Phi_{j}}{\partial n(\mathbf{x}) \partial n(\mathbf{y})}(\mathbf{x}, \mathbf{y}) f(\mathbf{y}) d s(\mathbf{y}) & & \mathbf{x} \in \Gamma, \\
& :=\left(N D_{j} f\right)(\mathbf{x}), & \\
\frac{\partial}{\partial \tau}\left(S_{j} f\right)(\mathbf{x}) & =\int_{\Gamma} \frac{\partial \Phi_{j}}{\partial \tau(\mathbf{x})}(\mathbf{x}, \mathbf{y}) f(\mathbf{y}) d s(\mathbf{y}) & \mathbf{x} \in \Gamma, &
\end{array}
$$




$$
\begin{array}{rlr}
\frac{\partial}{\partial \tau}\left(D_{j} f\right)(\mathbf{x}) & =\int_{\Gamma} \frac{\partial^{2} \Phi_{j}}{\partial \tau(\mathbf{x}) \partial n(\mathbf{y})}(\mathbf{x}, \mathbf{y}) f(\mathbf{y}) d s(\mathbf{y}) \pm \frac{1}{2} \frac{\partial f}{\partial \tau}(\mathbf{x}) & \\
& :=\left(T D_{j} f\right)(\mathbf{x}) \pm \frac{1}{2} \frac{\partial f}{\partial \tau}(\mathbf{x}), \quad \mathbf{x} \in \Gamma,
\end{array}
$$

where the upper (lower) sign indicates the limits obtained by approaching the boundary $\Gamma$ from $\Omega_{0}\left(\Omega_{1}\right)$. This means when $j=0,(j=1)$. For the last equation, we assume $f$ to be continuously differentiable. We presented the jump relations for continuous densities only for simplicity, since in the following proof we use a Sobolev space setting.

Here, we have to mention the continuity of the single-layer potentials in $\mathbb{R}^{2}$ and the discontinuity of the double-layer potentials $\left( \pm \frac{1}{2} f\right)$. All the integrals are well defined and particularly the potentials $S_{j}$, $D_{j}$ and $N S_{j}$ have weakly singular kernels (logarithmic singularity), the potentials $T S_{j}$ have Cauchy type singularity (of order $1 /|\mathbf{x}-\mathbf{y}|$ ) and the potentials $N D_{j}, T D_{j}$ are hypersingular (of order $1 /|\mathbf{x}-\mathbf{y}|^{2}$ ).

Theorem 3.2. If $\kappa_{1}^{2}$ is not an interior Dirichlet eigenvalue and $\kappa_{0}^{2}$ is not an interior Dirichlet and Neumann eigenvalue, then the problem (3.1)-(3.3) has a unique solution.

Proof. We apply the direct method, see for instance [9], to transform the problem into a system of integral equations. We consider Green's second theorem in the interior domain

$$
\begin{aligned}
-u_{1}(\mathbf{x})= & \int_{\Gamma} \frac{\partial \Phi_{1}}{\partial n(\mathbf{y})}(\mathbf{x}, \mathbf{y}) u_{1}(\mathbf{y}) d s(\mathbf{y}) \\
& -\int_{\Gamma} \Phi_{1}(\mathbf{x}, \mathbf{y}) \frac{\partial u_{1}}{\partial n(\mathbf{y})}(\mathbf{y}) d s(\mathbf{y}), \\
= & \left(D_{1} u_{1}\right)(\mathbf{x})-\left(S_{1} \partial_{\eta} u_{1}\right)(\mathbf{x}), \quad \mathbf{x} \in \Omega_{1},
\end{aligned}
$$

similarly

$$
-v_{1}(\mathbf{x})=\left(D_{1} v_{1}\right)(\mathbf{x})-\left(S_{1} \partial_{\eta} v_{1}\right)(\mathbf{x}), \quad \mathbf{x} \in \Omega_{1},
$$

and in the exterior domain

$$
\begin{array}{ll}
u_{0}(\mathbf{x})=\left(D_{0} u_{0}\right)(\mathbf{x})-\left(S_{0} \partial_{\eta} u_{0}\right)(\mathbf{x}), & \mathbf{x} \in \Omega_{0}, \\
v_{0}(\mathbf{x})=\left(D_{0} v_{0}\right)(\mathbf{x})-\left(S_{0} \partial_{\eta} v_{0}\right)(\mathbf{x}), & \mathbf{x} \in \Omega_{0} .
\end{array}
$$


Letting $\mathbf{x} \rightarrow \Gamma$, in the above formulas and taking the normal and the tangential derivatives on $\Gamma$, we obtain

$$
\begin{aligned}
\left(N S_{j} \pm \frac{1}{2} I\right) \partial_{\eta} u_{j} & =N D_{j} u_{j}, & \left(N S_{j} \pm \frac{1}{2} I\right) \partial_{\eta} v_{j} & =N D_{j} v_{j} \\
\left(D_{j} \mp \frac{1}{2} I\right) u_{j} & =S_{j} \partial_{\eta} u_{j}, & \left(D_{j} \mp \frac{1}{2} I\right) v_{j} & =S_{j} \partial_{\eta} v_{j} \\
T D_{j} u_{j}-T S_{j} \partial_{\eta} u_{j} & = \pm \frac{1}{2} \partial_{\tau} u_{j}, & T D_{j} v_{j}-T S_{j} \partial_{\eta} v_{j} & = \pm \frac{1}{2} \partial_{\tau} v_{j} .
\end{aligned}
$$

Combining the relations in (3.11b) for $j=0$ with the boundary conditions $(3.2 \mathrm{~d})$ and $(3.2 \mathrm{~b})$, respectively, we have

$$
\begin{aligned}
\left(D_{0}-\frac{1}{2} I\right) u_{0}= & \frac{\widetilde{\epsilon}_{1}}{\widetilde{\epsilon}_{0}} S_{0} \partial_{\eta} u_{1}-\frac{\beta_{1}}{\widetilde{\epsilon}_{0} \omega} S_{0} \partial_{\tau} v_{1} \\
& +\frac{\beta_{0}}{\widetilde{\epsilon}_{0} \omega} S_{0} \partial_{\tau} v_{0}-S_{0} \partial_{\eta} e_{3}^{\mathrm{inc}}, \\
\left(D_{0}-\frac{1}{2} I\right) v_{0}= & \frac{\widetilde{\mu}_{1}}{\widetilde{\mu}_{0}} S_{0} \partial_{\eta} v_{1}+\frac{\beta_{1}}{\widetilde{\mu}_{0} \omega} S_{0} \partial_{\tau} u_{1}-\frac{\beta_{0}}{\widetilde{\mu}_{0} \omega} S_{0} \partial_{\tau} u_{0}-\frac{\beta_{0}}{\widetilde{\mu}_{0} \omega} S_{0} \partial_{\tau} e_{3}^{\mathrm{inc}} .
\end{aligned}
$$

We define

$$
K_{j}:=\left(N S_{j} \pm \frac{1}{2} I\right)^{-1} N D_{j}, \quad L_{j}:=2\left(T D_{j}-T S_{j} K_{j}\right) .
$$

The operator $K_{0}$ is well defined since the integral equation (3.11a) for $j=0$ corresponds to the solution of the interior Neumann problem and we assumed $\kappa_{0}^{2}$ not to be an interior eigenvalue. Similarly, $K_{1}$ is well defined if $\kappa_{1}^{2}$ is not an interior Dirichlet eigenvalue.

Then, the system of equations (3.12), using (3.11), is transformed to

$$
\begin{array}{r}
\left(D_{0}-\frac{1}{2} I\right) u_{0}-\frac{\widetilde{\epsilon}_{1}}{\widetilde{\epsilon}_{0}} S_{0} K_{1} u_{1}-\frac{\beta_{1}}{\widetilde{\epsilon}_{0} \omega} S_{0} L_{1} v_{1}-\frac{\beta_{0}}{\widetilde{\epsilon}_{0} \omega} S_{0} L_{0} v_{0} \\
=-S_{0} \partial_{\eta} e_{3}^{\text {inc }}, \\
\left(D_{0}-\frac{1}{2} I\right) v_{0}-\frac{\widetilde{\mu}_{1}}{\widetilde{\mu}_{0}} S_{0} K_{1} v_{1}+\frac{\beta_{1}}{\widetilde{\mu}_{0} \omega} S_{0} L_{1} u_{1}+\frac{\beta_{0}}{\widetilde{\mu}_{0} \omega} S_{0} L_{0} u_{0} \\
=-\frac{\beta_{0}}{\widetilde{\mu}_{0} \omega} S_{0} \partial_{\tau} e_{3}^{\text {inc }} .
\end{array}
$$


We consider now equations (3.2a) and (3.2c), to obtain

$$
\begin{array}{r}
\left(D_{0}-\frac{1}{2} I\right) u_{0}-\frac{\widetilde{\epsilon}_{1}}{\widetilde{\epsilon}_{0}} S_{0} K_{1} u_{0}-\frac{\beta_{1}}{\widetilde{\epsilon}_{0} \omega} S_{0} L_{1} v_{0}-\frac{\beta_{0}}{\widetilde{\epsilon}_{0} \omega} S_{0} L_{0} v_{0} \\
=-S_{0} \partial_{\eta} e_{3}^{\mathrm{inc}}+\frac{\widetilde{\epsilon}_{1}}{\widetilde{\epsilon}_{0}} S_{0} K_{1} e_{3}^{\mathrm{inc}}, \\
\left(D_{0}-\frac{1}{2} I\right) v_{0}-\frac{\widetilde{\mu}_{1}}{\widetilde{\mu}_{0}} S_{0} K_{1} v_{0}+\frac{\beta_{1}}{\widetilde{\mu}_{0} \omega} S_{0} L_{1} u_{0}+\frac{\beta_{0}}{\widetilde{\mu}_{0} \omega} S_{0} L_{0} u_{0} \\
=-\frac{\beta_{0}}{\widetilde{\mu}_{0} \omega} S_{0} \partial_{\tau} e_{3}^{\mathrm{inc}}-\frac{\beta_{1}}{\widetilde{\mu}_{0} \omega} S_{0} L_{1} e_{3}^{\mathrm{inc}} .
\end{array}
$$

The above system in compact form reads

$$
(\mathbf{D}+\mathbf{K}) \mathbf{u}=\mathbf{b},
$$

where

$$
\begin{aligned}
\mathbf{D} & =\left(\begin{array}{cc}
D_{0}-1 / 2 I & 0 \\
0 & D_{0}-1 / 2 I
\end{array}\right), \\
\mathbf{K} & =\left(\begin{array}{cc}
-\left(\widetilde{\epsilon}_{1} / \widetilde{\epsilon}_{0}\right) S_{0} K_{1} & -\left(1 / \widetilde{\epsilon}_{0} \omega\right) S_{0}\left(\beta_{1} L_{1}+\beta_{0} L_{0}\right) \\
\left(1 / \widetilde{\mu}_{0} \omega\right) S_{0}\left(\beta_{1} L_{1}+\beta_{0} L_{0}\right) & -\left(\widetilde{\mu}_{1} / \widetilde{\mu}_{0}\right) S_{0} K_{1}
\end{array}\right), \\
\mathbf{u} & =\left(\begin{array}{l}
\left.u_{0}\right|_{\Gamma} \\
\left.v_{0}\right|_{\Gamma}
\end{array}\right), \\
\mathbf{b} & =\left(\begin{array}{c}
-S_{0} \partial_{\eta}+\left(\widetilde{\epsilon}_{1} / \widetilde{\epsilon}_{0}\right) S_{0} K_{1} \\
-\left(1 / \widetilde{\mu}_{0} \omega\right) S_{0}\left(\beta_{0} \partial_{\tau}+\beta_{1} L_{1}\right)
\end{array}\right) e_{3}^{\text {inc }} .
\end{aligned}
$$

We assume that $\Gamma$ is of class $C^{2, \alpha}, 0<\alpha \leq 1$. We know that $D_{0}: H^{-1 / 2}(\Gamma) \rightarrow H^{-1 / 2}(\Gamma)$ is compact; thus, $\left(D_{0}-1 / 2 I\right)^{-1}$ : $H^{-1 / 2}(\Gamma) \rightarrow H^{-1 / 2}(\Gamma)$ is bounded, if $\kappa_{0}^{2}$ is not an interior Dirichlet eigenvalue. Then, D : $\left(H^{-1 / 2}(\Gamma)\right)^{2} \rightarrow\left(H^{-1 / 2}(\Gamma)\right)^{2}$ is bounded and (3.14) is transformed to

$$
\left(\mathbf{I}+\mathbf{D}^{-1} \mathbf{K}\right) \mathbf{u}=\mathbf{D}^{-1} \mathbf{b} .
$$

First, we show that $\mathbf{K}$ is compact. We recall that

$$
\begin{aligned}
S_{0} K_{1} & =S_{0}\left(N S_{1}-\frac{1}{2} I\right)^{-1} N D_{1} \\
S_{0}\left(\beta_{1} L_{1}+\beta_{0} L_{0}\right) & =2 S_{0}\left(\beta_{1} T D_{1}+\beta_{0} T D_{0}-\beta_{0} T S_{0}\left(N S_{0}+\frac{1}{2} I\right)^{-1} N D_{0}\right)
\end{aligned}
$$




$$
-2 \beta_{1} S_{0} T S_{1}\left(N S_{1}-\frac{1}{2} I\right)^{-1} N D_{1},
$$

and the following properties, see $[\mathbf{4}, \mathbf{5}], S_{0}: H^{-1 / 2}(\Gamma) \rightarrow H^{-1 / 2}(\Gamma)$ is compact, $N D_{j}, T D_{j}: H^{1 / 2}(\Gamma) \rightarrow H^{-1 / 2}(\Gamma)$ are bounded, $T S_{j}$ : $H^{-1 / 2}(\Gamma) \rightarrow H^{-1 / 2}(\Gamma)$ are bounded and $\left(N S_{j} \pm \frac{1}{2} I\right)^{-1}: H^{-1 / 2}(\Gamma) \rightarrow$ $H^{-1 / 2}(\Gamma)$ are bounded due to compactness of $N S_{j}: H^{-1 / 2}(\Gamma) \rightarrow$ $H^{-1 / 2}(\Gamma)$. Then, the operators

$$
S_{0} K_{1}, S_{0}\left(\beta_{1} L_{1}+\beta_{0} L_{0}\right): H^{1 / 2}(\Gamma) \longrightarrow H^{-1 / 2}(\Gamma),
$$

are compact. Hence, $\mathbf{K}:\left(H^{1 / 2}(\Gamma)\right)^{2} \rightarrow\left(H^{-1 / 2}(\Gamma)\right)^{2}$ is also compact resulting in the compactness of $\mathbf{D}^{-1} \mathbf{K}:\left(H^{1 / 2}(\Gamma)\right)^{2} \rightarrow\left(H^{-1 / 2}(\Gamma)\right)^{2}$.

Next we prove the uniqueness of solutions of equation (3.15). Solvability follows from the Fredholm alternative theorem. Let $\left(\phi_{0}, \psi_{0}\right)^{T}$ be the solution of the homogeneous form of (3.15). Then, the potentials

$$
\begin{array}{ll}
u_{0,0}(\mathbf{x})=\left(D_{0} \phi_{0}\right)(\mathbf{x})-\left(S_{0} \partial_{\eta} \phi_{0}\right)(\mathbf{x}), & \mathbf{x} \in \Omega_{0}, \\
v_{0,0}(\mathbf{x})=\left(D_{0} \psi_{0}\right)(\mathbf{x})-\left(S_{0} \partial_{\eta} \psi_{0}\right)(\mathbf{x}), & \mathbf{x} \in \Omega_{0},
\end{array}
$$

and

$$
\begin{array}{ll}
u_{1,1}(\mathbf{x})=-\left(D_{1} \phi_{1}\right)(\mathbf{x})+\left(S_{1} \partial_{\eta} \phi_{1}\right)(\mathbf{x}), & \mathbf{x} \in \Omega_{1}, \\
v_{1,1}(\mathbf{x})=-\left(D_{1} \psi_{1}\right)(\mathbf{x})+\left(S_{1} \partial_{\eta} \psi_{1}\right)(\mathbf{x}), & \mathbf{x} \in \Omega_{1},
\end{array}
$$

solve the homogeneous form of the problem (3.1)-(3.3). From Theorem 3.1, we have that $u_{0,0}=v_{0,0}=0$ in $\Omega_{0}$ and $u_{1,1}=v_{1,1}=0$ in $\Omega_{1}$ where the densities $\phi_{1}, \psi_{1}$ depend on the solution of the homogeneous case.

We construct

$$
\begin{array}{ll}
u_{0,1}(\mathbf{x})=\left(D_{0} \phi_{0}\right)(\mathbf{x})-\left(S_{0} \partial_{\eta} \phi_{0}\right)(\mathbf{x}), & \mathbf{x} \in \Omega_{1}, \\
v_{0,1}(\mathbf{x})=\left(D_{0} \psi_{0}\right)(\mathbf{x})-\left(S_{0} \partial_{\eta} \psi_{0}\right)(\mathbf{x}), & \mathbf{x} \in \Omega_{1},
\end{array}
$$

and

$$
\begin{array}{ll}
u_{1,0}(\mathbf{x})=-\left(D_{1} \phi_{1}\right)(\mathbf{x})+\left(S_{1} \partial_{\eta} \phi_{1}\right)(\mathbf{x}), & \mathbf{x} \in \Omega_{0}, \\
v_{1,0}(\mathbf{x})=-\left(D_{1} \psi_{1}\right)(\mathbf{x})+\left(S_{1} \partial_{\eta} \psi_{1}\right)(\mathbf{x}), & \mathbf{x} \in \Omega_{0} .
\end{array}
$$

Considering the jump relations, at the boundary $\Gamma$ we obtain

$$
\begin{aligned}
u_{0,0}-u_{0,1}=\phi_{0}, & u_{1,1}-u_{1,0}=\phi_{1}, \\
v_{0,0}-v_{0,1}=\psi_{0}, & v_{1,1}-v_{1,0}=\psi_{1} .
\end{aligned}
$$


Since $u_{0,0}=u_{1,1}=v_{0,0}=v_{1,1}=0, \phi_{0}=\phi_{1}$ and $\psi_{0}=\psi_{1}$, on $\Gamma$, we find

$$
u_{0,1}=u_{1,0}, \quad v_{0,1}=v_{1,0}, \quad \mathbf{x} \in \Gamma .
$$

Similarly, we can rewrite the other two boundary conditions of (3.4) for those fields taking the differences of the normal and tangential derivatives as $\mathbf{x} \rightarrow \Gamma$. Thus, we see that $u_{0,1}, v_{0,1}, u_{1,0}$ and $v_{1,0}$ solve the homogeneous problem, but with $\kappa_{1}$ and $\kappa_{0}$ interchanged and from Theorem 3.1, we also get $u_{0,1}=v_{0,1}=0$, on $\Gamma$ and hence $\phi_{0}=\psi_{0}=0$ from (3.16).

In order to handle the hypersingularity of the operators $T D_{j}$ we work in a similar way as Mitzner [15] derived the Maue's formula [14] of the hypersingular operator $N D_{j}$, namely

$$
\left(N D_{j} f\right)(\mathbf{x})=\left(T S_{j} \frac{\partial f}{\partial \tau}\right)(\mathbf{x})+\kappa_{j}^{2} \widehat{\boldsymbol{n}}(\mathbf{x}) \cdot\left(S_{j} \widehat{\boldsymbol{n}} f\right)(\mathbf{x}), \quad \mathbf{x} \in \Gamma .
$$

This transformation reduces the hypersingularity to singularity of Cauchy type (first term) and to a weak singularity (second term).

Theorem 3.3. Let $f \in C^{1, \alpha}(\Gamma)$. The hypersingular operator $T D_{j}$ can be transformed to

$$
\left(T D_{j} f\right)(\boldsymbol{x})=-\left(N S_{j} \frac{\partial f}{\partial \tau}\right)(\boldsymbol{x})+\kappa_{j}^{2} \widehat{\boldsymbol{\tau}}(\boldsymbol{x}) \cdot\left(S_{j} \widehat{\boldsymbol{n}} f\right)(\boldsymbol{x}), \quad \boldsymbol{x} \in \Gamma
$$

Proof. We recall equation (3.9). Applying Green's first theorem to $\mathbf{v} \cdot \nabla u_{1}, \mathbf{v}$ arbitrary constant vector, and $\Phi_{1}$, using that

$$
\Delta \Phi_{1}(\mathbf{x}, \mathbf{y})+\kappa_{1}^{2} \Phi_{1}(\mathbf{x}, \mathbf{y})=-\delta(\mathbf{x}-\mathbf{y}),
$$

yields

$$
\begin{aligned}
\int_{\Omega_{1}} \nabla_{y}(\mathbf{v} & \left.\cdot \nabla_{y} u_{1}(\mathbf{y})\right) \cdot \nabla_{y} \Phi_{1}(\mathbf{x}, \mathbf{y}) d \mathbf{y} \\
& -\kappa_{1}^{2} \int_{\Omega_{1}} \Phi_{1}(\mathbf{x}, \mathbf{y}) \mathbf{v} \cdot \nabla_{y} u_{1}(\mathbf{y}) d \mathbf{y} \\
= & \mathbf{v} \cdot \nabla_{x} u_{1}(\mathbf{x})+\int_{\Gamma} \frac{\partial \Phi_{1}}{\partial n(\mathbf{y})}(\mathbf{x}, \mathbf{y}) \mathbf{v} \cdot \nabla_{y} u_{1}(\mathbf{y}) d s(\mathbf{y})
\end{aligned}
$$


The first integral can be transformed to

$$
\begin{aligned}
& \int_{\Omega_{1}} \nabla\left(\mathbf{v} \cdot \nabla u_{1}\right) \cdot \nabla \Phi_{1} d \mathbf{y} \\
&=-\int_{\Omega_{1}}(\left.\kappa_{1}^{2} u_{1} \mathbf{v}+\nabla \times\left(\mathbf{v} \times \nabla u_{1}\right)\right) \cdot \nabla \Phi_{1} d \mathbf{y} \\
&=-\kappa_{1}^{2} \mathbf{v} \cdot \int_{\Omega_{1}} u_{1} \nabla \Phi_{1} d \mathbf{y} \\
& \quad-\mathbf{v} \cdot \int_{\Gamma}\left(\widehat{\boldsymbol{n}} \times \nabla \Phi_{1}\right) \times \nabla u_{1} d s(\mathbf{y}) .
\end{aligned}
$$

Then, (3.19) reads

$$
\begin{aligned}
\mathbf{v} \cdot\left(\kappa_{1}^{2} \int_{\Omega_{1}} \nabla_{y}\left(\Phi_{1} u_{1}\right) d \mathbf{y}+\int_{\Gamma}\left(\widehat{\boldsymbol{n}} \times \nabla_{y} \Phi_{1}\right) \times \nabla_{y} u_{1} d s(\mathbf{y})\right. \\
\left.+\int_{\Gamma} \frac{\partial \Phi_{1}}{\partial n_{y}} \nabla_{y} u_{1} d s(\mathbf{y})\right)=-\mathbf{v} \cdot \nabla_{x} u_{1} .
\end{aligned}
$$

Using some vector identities and suppressing the inner products with $\mathbf{v}$ (holds for any vector), we end up to

$-\nabla_{x} u_{1}=\int_{\Gamma}\left(-\nabla_{y} \Phi_{1} \times\left(\widehat{\boldsymbol{n}}_{y} \times \nabla_{y} u_{1}\right)+\kappa_{1}^{2} \Phi_{1} u_{1} \widehat{\boldsymbol{n}}_{y}+\nabla_{y} \Phi_{1} \frac{\partial u_{1}}{\partial n_{y}}\right) d s(\mathbf{y})$,

for $\mathbf{x} \in \Omega_{1}$. We multiply this equation with $\widehat{\boldsymbol{n}}(\mathbf{x})$ (inner product) and consider the limit as $\mathbf{x}$ approaches the boundary $\Gamma$ from inside and the corresponding jump relations. We obtain [15]

$$
\begin{aligned}
- & \frac{1}{2} \frac{\partial}{\partial n} u_{1} \\
= & \int_{\Gamma}\left(\left(\widehat{\boldsymbol{n}}_{x} \times \nabla_{x} \Phi_{1}\right) \cdot\left(\widehat{\boldsymbol{n}}_{y} \times \nabla_{y} u_{1}\right)+\kappa_{1}^{2} \Phi_{1} u_{1}\left(\widehat{\boldsymbol{n}}_{x} \cdot \widehat{\boldsymbol{n}}_{y}\right)\right) d s(\mathbf{y}) \\
& -\int_{\Gamma} \frac{\partial \Phi_{1}}{\partial n_{x}} \frac{\partial u_{1}}{\partial n_{y}} d s(\mathbf{y}), \quad \mathbf{x} \in \Gamma .
\end{aligned}
$$

Now, equating the above equation and the normal derivative of (3.9) as $\mathbf{x} \rightarrow \Gamma$, we obtain (3.17) in $\mathbb{R}^{2}$. 
We take the tangential derivative of (3.9), and, considering the jump relations, we get

$$
-\frac{\partial}{\partial \tau} u_{1}(\mathbf{x})=\left(T D_{1} u_{1}\right)(\mathbf{x})-\frac{1}{2} \frac{\partial u_{1}}{\partial \tau}(\mathbf{x})-\left(T S_{1} \frac{\partial u_{1}}{\partial n}\right)(\mathbf{x}), \mathbf{x} \in \Gamma .
$$

We replace $\widehat{\boldsymbol{n}}(\mathbf{x})$ by $\widehat{\boldsymbol{\tau}}(\mathbf{x})$ in (3.20) (considering the appropriate jump relations) and restricting ourselves in $\mathbb{R}^{2}$, we have

$$
\begin{aligned}
-\frac{1}{2} \frac{\partial}{\partial \tau} u_{1}= & \int_{\Gamma}\left(-\left(\widehat{\boldsymbol{n}}_{x} \cdot \nabla_{x} \Phi_{1}\right)\left(\widehat{\boldsymbol{\tau}}_{y} \cdot \nabla_{y} u_{1}\right)+\kappa_{1}^{2} \Phi_{1} u_{1}\left(\widehat{\boldsymbol{\tau}}_{x} \cdot \widehat{\boldsymbol{n}}_{y}\right)\right) d s(\mathbf{y}) \\
& -\int_{\Gamma} \frac{\partial \Phi_{1}}{\partial \tau_{x}} \frac{\partial u_{1}}{\partial n_{y}} d s(\mathbf{y}), \quad \mathbf{x} \in \Gamma .
\end{aligned}
$$

Observing the last two equations, we obtain (3.18), the equivalent of the Maue's formula for the tangential derivative of the double-layer potential which also reduces the hypersingularity of the potential.

4. Numerical results. In this section, we present numerical examples by implementing the proposed method. We use quadrature rules to integrate the singularities considering trigonometric interpolation. Regarding the convergence and the error analysis of the quadrature formulas, we refer the reader to [11] for the weakly singular operators and to [10] for the hypersingular. We solve the system of integral equations considering these rules by the Nyström method.

We assume the following parametrization for the boundary:

$$
\Gamma=\left\{\mathbf{z}(t)=\left(z_{1}(t), z_{2}(t)\right): t \in[0,2 \pi]\right\},
$$

where $\mathbf{z}: \mathbb{R} \rightarrow \mathbb{R}^{2}$ is a $C^{2}$-smooth, $2 \pi$-periodic and counterclockwise oriented parametrization. We assume, in addition, that $\mathbf{z}$ is injective in $[0,2 \pi)$, that is, $\mathbf{z}^{\prime}(t) \neq 0$ for all $t \in[0,2 \pi]$.

Now, we transform the operators in (3.7) and their derivatives, see (3.8), into their parametric forms

$$
\begin{aligned}
& \left(S_{j} \psi\right)(t)=\int_{0}^{2 \pi} M^{S_{j}}(t, s) \psi(s) d s \\
& \left(D_{j} \psi\right)(t)=\int_{0}^{2 \pi} M^{D_{j}}(t, s) \psi(s) d s
\end{aligned}
$$




$$
\begin{aligned}
& \left(N S_{j} \psi\right)(t)=\int_{0}^{2 \pi} M^{N S_{j}}(t, s) \psi(s) d s, \\
& \left(T S_{j} \psi\right)(t)=\int_{0}^{2 \pi} M^{T S_{j}}(t, s) \psi(s) d s,
\end{aligned}
$$

and the special forms

$$
\begin{aligned}
\left(N D_{j} \psi\right)(t)= & \frac{1}{\left|\mathbf{z}^{\prime}(t)\right|} \int_{0}^{2 \pi}\left[\frac{1}{4 \pi} \cot \left(\frac{s-t}{2}\right) \frac{\partial \psi}{\partial s}(s)-M^{N D_{j}}(t, s) \psi(s)\right] d s \\
& +\kappa_{j}^{2} \int_{0}^{2 \pi}(\widehat{\boldsymbol{n}}(t) \cdot \widehat{\boldsymbol{n}}(s)) M^{S_{j}}(t, s) \psi(s) d s,
\end{aligned}
$$

$$
\begin{aligned}
\left(T D_{j} \psi\right)(t)= & \frac{1}{\left|\mathbf{z}^{\prime}(t)\right|} \int_{0}^{2 \pi} M^{T D_{j}}(t, s) \psi(s) d s \\
& +\kappa_{j}^{2} \int_{0}^{2 \pi}(\widehat{\boldsymbol{\tau}}(t) \cdot \widehat{\boldsymbol{n}}(s)) M^{S_{j}}(t, s) \psi(s) d s,
\end{aligned}
$$

for $t \in[0,2 \pi], j=0,1$ and $\psi(t)=f(\mathbf{z}(t)), \widehat{\boldsymbol{n}}(t)=\widehat{\boldsymbol{n}}(\mathbf{z}(t)), \widehat{\boldsymbol{\tau}}(t)=$ $\widehat{\boldsymbol{\tau}}(\mathbf{z}(t))$, where

$$
\begin{aligned}
M^{S_{j}}(t, s)= & \frac{i}{4} H_{0}^{(1)}\left(\kappa_{j}|\mathbf{r}(t, s)|\right)\left|\mathbf{z}^{\prime}(s)\right|, \\
M^{D_{j}}(t, s)= & \frac{i \kappa_{j}}{4} \frac{\widehat{\boldsymbol{n}}(s) \cdot \mathbf{r}(t, s)}{|\mathbf{r}(t, s)|} H_{1}^{(1)}\left(\kappa_{j}|\mathbf{r}(t, s)|\right)\left|\mathbf{z}^{\prime}(s)\right|, \\
M^{N S_{j}}(t, s)= & -\frac{i \kappa_{j}}{4} \frac{\widehat{\boldsymbol{n}}(t) \cdot \mathbf{r}(t, s)}{|\mathbf{r}(t, s)|} H_{1}^{(1)}\left(\kappa_{j}|\mathbf{r}(t, s)|\right)\left|\mathbf{z}^{\prime}(s)\right|, \\
M^{T S_{j}}(t, s)= & -\frac{i \kappa_{j}}{4} \frac{\widehat{\boldsymbol{\tau}}(t) \cdot \mathbf{r}(t, s)}{|\mathbf{r}(t, s)|} H_{1}^{(1)}\left(\kappa_{j}|\mathbf{r}(t, s)|\right)\left|\mathbf{z}^{\prime}(s)\right|, \\
M^{N D_{j}}(t, s)= & \frac{i}{4} M(t, s)\left[\kappa_{j}^{2} H_{0}^{(1)}\left(\kappa_{j}|\mathbf{r}(t, s)|\right)-2 \kappa_{j} \frac{H_{1}^{(1)}\left(\kappa_{j}|\mathbf{r}(t, s)|\right)}{|\mathbf{r}(t, s)|}\right] \\
& +\frac{i \kappa_{j}}{4} \frac{\mathbf{z}^{\prime}(t) \cdot \mathbf{z}^{\prime}(s)}{|\mathbf{r}(t, s)|} H_{1}^{(1)}\left(\kappa_{j}|\mathbf{r}(t, s)|\right)+\frac{1}{8 \pi} \sin ^{-2}\left(\frac{t-s}{2}\right), \\
M^{T D_{j}}(t, s)= & \frac{i}{4} M^{J}(t, s)\left[\kappa_{j}^{2} H_{0}^{(1)}\left(\kappa_{j}|\mathbf{r}(t, s)|\right)-2 \kappa_{j} \frac{H_{1}^{(1)}\left(\kappa_{j}|\mathbf{r}(t, s)|\right)}{|\mathbf{r}(t, s)|}\right]
\end{aligned}
$$




$$
+\frac{i \kappa_{j}}{4} \frac{\mathbf{J ~ z}^{\prime}(t) \cdot \mathbf{z}^{\prime}(s)}{|\mathbf{r}(t, s)|} H_{1}^{(1)}\left(\kappa_{j}|\mathbf{r}(t, s)|\right),
$$

with $\mathbf{r}(t, s)=\mathbf{z}(t)-\mathbf{z}(s)$, and

$$
\begin{aligned}
M(t, s) & =\frac{\left(\mathbf{z}^{\prime}(t) \cdot \mathbf{r}(t, s)\right)\left(\mathbf{z}^{\prime}(s) \cdot \mathbf{r}(t, s)\right)}{|\mathbf{r}(t, s)|^{2}}, \\
M^{J}(t, s) & =\frac{\left(\mathbf{J ~ z ~ z}^{\prime}(t) \cdot \mathbf{r}(t, s)\right)\left(\mathbf{z}^{\prime}(s) \cdot \mathbf{r}(t, s)\right)}{|\mathbf{r}(t, s)|^{2}} .
\end{aligned}
$$

Here, we have used the formulas $H_{1}^{(1)}(t)=-H_{0}^{(1)^{\prime}}(t)$ and $H_{1}^{(1)^{\prime}}(t)=$ $H_{0}^{(1)}(t)-(1 / t) H_{1}^{(1)}(t)$. The form in equation (4.2a) is based on (3.17), derived by Kress [10] and improved in [12]. The derivation of $(4.2 \mathrm{~b})$ is easier. Namely, we define $\partial_{t_{J}}:=\mathbf{J ~ z ~ z}^{\prime} \partial_{z}$ and

$$
M^{T D_{j}}(t, s):=\frac{\partial^{2}}{\partial t_{J} \partial s} M^{S_{j}}(t, s) .
$$

Then

$$
\begin{aligned}
-\left(N S_{j} \frac{\partial f}{\partial \tau}\right)(\mathbf{z}(t))= & -\int_{0}^{2 \pi} \frac{\mathbf{J} \mathbf{z}^{\prime}(t)}{\left|\mathbf{z}^{\prime}(t)\right|} \frac{\partial}{\partial z(t)} M^{S_{j}}(t, s) \\
& \cdot \frac{\mathbf{z}^{\prime}(s)}{\left|\mathbf{z}^{\prime}(s)\right|} \frac{\partial \psi}{\partial z(s)}(s)\left|\mathbf{z}^{\prime}(s)\right| d s \\
= & -\frac{1}{\left|\mathbf{z}^{\prime}(t)\right|} \int_{0}^{2 \pi} \frac{\partial}{\partial t_{J}} M^{S_{j}}(t, s) \frac{\partial}{\partial s} \psi(s) d s \\
= & \frac{1}{\left|\mathbf{z}^{\prime}(t)\right|} \int_{0}^{2 \pi} \frac{\partial^{2}}{\partial t_{J} \partial s} M^{S_{j}}(t, s) \psi(s) d s,
\end{aligned}
$$

and (4.2b) follows by simply adding the parametrized form of the second term in the right-hand side of (3.18). The kernels in (4.3) admit the decomposition

$$
M^{k}(t, s)=M_{1}^{k}(t, s) \ln \left(4 \sin ^{2}\left(\frac{t-s}{2}\right)\right)+M_{2}^{k}(t, s),
$$

for $k=S_{j}, D_{j}, N S_{j}, N D_{j}, T D_{j}$ where $M_{1}^{k}$ and $M_{2}^{k}$ are analytic, due to logarithmic singularity of the functions at $t=s$. The case of $M^{T S_{j}}$ has to be treated differently because of the Cauchy type singularity of 
the kernel as $t=s$. Thus, we split the kernel as

$$
\begin{aligned}
M^{T S_{j}}(t, s)= & M_{1}^{T S_{j}}(t, s) \ln \left(4 \sin ^{2}\left(\frac{t-s}{2}\right)\right)+\frac{1}{4 \pi} \cot \left(\frac{s-t}{2}\right) \\
& +M_{2}^{T S_{j}}(t, s) .
\end{aligned}
$$

The kernels $M_{1}^{k}$ are defined for $t \neq s$ by, see [10]

$$
\begin{aligned}
M_{1}^{S_{j}}(t, s)= & -\frac{1}{4 \pi} J_{0}\left(\kappa_{j}|\mathbf{r}(t, s)|\right)\left|\mathbf{z}^{\prime}(s)\right|, \\
M_{1}^{D_{j}}(t, s)= & -\frac{\kappa_{j}}{4 \pi} \frac{\widehat{\boldsymbol{n}}(s) \cdot \mathbf{r}(t, s)}{|\mathbf{r}(t, s)|} J_{1}\left(\kappa_{j}|\mathbf{r}(t, s)|\right)\left|\mathbf{z}^{\prime}(s)\right|, \\
M_{1}^{N S_{j}}(t, s)= & \frac{\kappa_{j}}{4 \pi} \frac{\widehat{\boldsymbol{n}}(t) \cdot \mathbf{r}(t, s)}{|\mathbf{r}(t, s)|} J_{1}\left(\kappa_{j}|\mathbf{r}(t, s)|\right)\left|\mathbf{z}^{\prime}(s)\right|, \\
M_{1}^{T S_{j}}(t, s)= & \frac{\kappa_{j}}{4 \pi} \frac{\widehat{\boldsymbol{\tau}}(t) \cdot \mathbf{r}(t, s)}{|\mathbf{r}(t, s)|} J_{1}\left(\kappa_{j}|\mathbf{r}(t, s)|\right)\left|\mathbf{z}^{\prime}(s)\right|, \\
M_{1}^{N D_{j}}(t, s)= & -\frac{1}{4 \pi} M(t, s)\left[\kappa_{j}^{2} J_{0}\left(\kappa_{j}|\mathbf{r}(t, s)|\right)-2 \kappa_{j} \frac{J_{1}\left(\kappa_{j}|\mathbf{r}(t, s)|\right)}{|\mathbf{r}(t, s)|}\right] \\
& -\frac{\kappa_{j}}{4 \pi} \frac{\mathbf{z}^{\prime}(t) \cdot \mathbf{z}^{\prime}(s)}{|\mathbf{r}(t, s)|} J_{1}\left(\kappa_{j}|\mathbf{r}(t, s)|\right), \\
M_{1}^{T D_{j}}(t, s)= & -\frac{1}{4 \pi} M^{J}(t, s)\left[\kappa_{j}^{2} J_{0}\left(\kappa_{j}|\mathbf{r}(t, s)|\right)-2 \kappa_{j} \frac{J_{1}\left(\kappa_{j}|\mathbf{r}(t, s)|\right)}{|\mathbf{r}(t, s)|}\right] \\
& -\frac{\kappa_{j}}{4 \pi} \frac{\mathbf{J}^{\prime}(t) \cdot \mathbf{z}^{\prime}(s)}{|\mathbf{r}(t, s)|} J_{1}\left(\kappa_{j}|\mathbf{r}(t, s)|\right),
\end{aligned}
$$

with diagonal terms

$$
\begin{aligned}
M_{1}^{S_{j}}(t, t) & =-\frac{1}{4 \pi}\left|\mathbf{z}^{\prime}(t)\right|, & M_{1}^{D_{j}}(t, t) & =0, \\
M_{1}^{N S_{j}}(t, t) & =0, & M_{1}^{T S_{j}}(t, t) & =0, \\
M_{1}^{N D_{j}}(t, t) & =-\frac{\kappa_{j}^{2}}{8 \pi}\left|\mathbf{z}^{\prime}(t)\right|^{2}, & M_{1}^{T D_{j}}(t, t) & =0,
\end{aligned}
$$

where $J_{0}$ and $J_{1}$ are the Bessel functions of order zero and one, respectively. The kernels $M_{2}^{k}$, for $t \neq s$, are given by

$$
M_{2}^{k}(t, s)=M^{k}(t, s)-M_{1}^{k}(t, s) \ln \left(4 \sin ^{2}\left(\frac{t-s}{2}\right)\right)
$$


and

$$
\begin{aligned}
M_{2}^{T S_{j}}(t, s) & =M^{T S_{j}}(t, s)-M_{1}^{T S_{j}}(t, s) \ln \left(4 \sin ^{2}\left(\frac{t-s}{2}\right)\right) \\
& -\frac{1}{4 \pi} \cot \left(\frac{s-t}{2}\right)
\end{aligned}
$$

with diagonal terms

$$
\begin{aligned}
M_{2}^{S_{j}}(t, t)= & {\left[\frac{i}{4}-\frac{C}{2 \pi}-\frac{1}{2 \pi} \ln \left(\frac{\kappa_{j}}{2}\left|\mathbf{z}^{\prime}(t)\right|\right)\right]\left|\mathbf{z}^{\prime}(t)\right|, } \\
M_{2}^{D_{j}}(t, t)= & \frac{1}{4 \pi} \frac{\widehat{\boldsymbol{n}}(t) \cdot \mathbf{z}^{\prime \prime}(t)}{\left|\mathbf{z}^{\prime}(t)\right|}, \\
M_{2}^{N S_{j}}(t, t)= & \frac{1}{4 \pi} \frac{\widehat{\boldsymbol{n}}(t) \cdot \mathbf{z}^{\prime \prime}(t)}{\left|\mathbf{z}^{\prime}(t)\right|}, \\
M_{2}^{T S_{j}}(t, t)= & -\frac{1}{4 \pi} \frac{\widehat{\boldsymbol{\tau}}(t) \cdot \mathbf{z}^{\prime \prime}(t)}{\left|\mathbf{z}^{\prime}(t)\right|}, \\
M_{2}^{N D_{j}}(t, t)= & {\left[\pi i-1-2 C-2 \ln \left(\frac{\kappa_{j}}{2}\left|\mathbf{z}^{\prime}(t)\right|\right)\right] \frac{\kappa_{j}^{2}}{8 \pi}\left|\mathbf{z}^{\prime}(t)\right|^{2}+\frac{1}{24 \pi} } \\
& +\frac{\left(\mathbf{z}^{\prime}(t) \cdot \mathbf{z}^{\prime \prime}(t)\right)^{2}}{4 \pi\left|\mathbf{z}^{\prime}(t)\right|^{4}}-\frac{\mathbf{z}^{\prime}(t) \cdot \mathbf{z}^{\prime \prime \prime}(t)}{12 \pi\left|\mathbf{z}^{\prime}(t)\right|^{2}}-\frac{\left|\mathbf{z}^{\prime \prime}(t)\right|^{2}}{8 \pi\left|\mathbf{z}^{\prime}(t)\right|^{2}}, \\
M_{2}^{T D_{j}}(t, t)= & -\frac{\left(\mathbf{z}^{\prime}(t) \cdot \mathbf{z}^{\prime \prime}(t)\right)\left(\mathbf{J} \mathbf{z}^{\prime}(t) \cdot \mathbf{z}^{\prime \prime}(t)\right)}{4 \pi\left|\mathbf{z}^{\prime}(t)\right|^{4}}+\frac{\mathbf{J} \mathbf{z}^{\prime}(t) \cdot \mathbf{z}^{\prime \prime \prime}(t)}{12 \pi\left|\mathbf{z}^{\prime}(t)\right|^{2}},
\end{aligned}
$$

where $C$ is the Euler's constant. For the last approximation, we used the same arguments as in the case of $M_{2}^{N D_{j}}$.

Considering the equidistant points $t_{j}=j \pi / n, j=0, \ldots, 2 n-1$, we use the trapezoidal rule to approximate the operators with smooth kernel

$$
\int_{0}^{2 \pi} \psi(s) d s \approx \frac{\pi}{n} \sum_{j=0}^{2 n-1} \psi\left(t_{j}\right),
$$


and the following quadrature rules for the singular kernels

$$
\begin{aligned}
\int_{0}^{2 \pi} \ln \left(4 \sin ^{2}\left(\frac{t-s}{2}\right)\right) \psi(s) d s & \approx \sum_{j=0}^{2 n-1} R_{j}^{(n)}(t) \psi\left(t_{j}\right), \\
\frac{1}{4 \pi} \int_{0}^{2 \pi} \cot \left(\frac{s-t}{2}\right) \frac{\partial}{\partial s} \psi(s) d s & \approx \sum_{j=0}^{2 n-1} T_{j}^{(n)}(t) \psi\left(t_{j}\right), \\
\int_{0}^{2 \pi} \cot \left(\frac{s-t}{2}\right) \psi(s) d s & \approx \sum_{j=0}^{2 n-1} S_{j}^{(n)}(t) \psi\left(t_{j}\right),
\end{aligned}
$$

with weights

$$
\begin{aligned}
& R_{j}^{(n)}(t)=-\frac{2 \pi}{n} \sum_{m=1}^{n-1} \frac{1}{m} \cos \left(m\left(t-t_{j}\right)\right)-\frac{\pi}{n^{2}} \cos \left(n\left(t-t_{j}\right)\right), \\
& T_{j}^{(n)}(t)=-\frac{1}{2 n} \sum_{m=1}^{n-1} m \cos \left(m\left(t-t_{j}\right)\right)-\frac{1}{4} \cos \left(n\left(t-t_{j}\right)\right), \\
& S_{j}^{(n)}(t)=\frac{\pi}{n}\left[1-(-1)^{j} \cos (n t)\right] \cot \left(\frac{t_{j}-t}{2}\right), \quad t \neq t_{j} .
\end{aligned}
$$

Then, the system (3.14), or similarly (3.15), considering the above parametric forms of the integral operators and the quadrature rules, is transformed to a linear system by applying the Nyström method.

To illustrate the efficiency of our method, we consider two different cases. In the first example, motivated by [23], we construct a model where the scattered fields can be analytically computed and in the second one we consider the scattering of obliquely incident waves.

In both examples, the parametrization of the obstacle is given by

$$
\mathbf{z}(t)=(2 \cos t+1.5 \cos 2 t-1,2.5 \sin t), \quad t \in[0,2 \pi] .
$$

4.1. Example with analytic solution. We consider four arbitrary points $\mathbf{z}_{1}, \mathbf{z}_{2} \in \Omega_{1}$ and $\mathbf{z}_{3}, \mathbf{z}_{4} \in \Omega_{0}$, and we define the boundary functions $f_{k}, k=1,2,3,4$ by

$$
f_{1}=H_{0}^{(1)}\left(\kappa_{1}\left|\mathbf{r}_{3}(\mathbf{x})\right|\right)-H_{0}^{(1)}\left(\kappa_{0}\left|\mathbf{r}_{1}(\mathbf{x})\right|\right),
$$




$$
\begin{aligned}
f_{2}= & -\widetilde{\mu}_{1} \omega \kappa_{1} H_{1}^{(1)}\left(\kappa_{1}\left|\mathbf{r}_{4}(\mathbf{x})\right|\right) \frac{\widehat{\boldsymbol{n}}(\mathbf{x}) \cdot \mathbf{r}_{4}(\mathbf{x})}{\left|\mathbf{r}_{4}(\mathbf{x})\right|} \\
& -\beta_{1} \kappa_{1} H_{1}^{(1)}\left(\kappa_{1}\left|\mathbf{r}_{3}(\mathbf{x})\right|\right) \frac{\widehat{\boldsymbol{\tau}}(\mathbf{x}) \cdot \mathbf{r}_{3}(\mathbf{x})}{\left|\mathbf{r}_{3}(\mathbf{x})\right|} \\
& +\widetilde{\mu}_{0} \omega \kappa_{0} H_{1}^{(1)}\left(\kappa_{0}\left|\mathbf{r}_{2}(\mathbf{x})\right|\right) \frac{\widehat{\boldsymbol{n}}(\mathbf{x}) \cdot \mathbf{r}_{2}(\mathbf{x})}{\left|\mathbf{r}_{2}(\mathbf{x})\right|} \\
& +\beta_{0} \kappa_{0} H_{1}^{(1)}\left(\kappa_{0}\left|\mathbf{r}_{1}(\mathbf{x})\right|\right) \frac{\widehat{\boldsymbol{\tau}}(\mathbf{x}) \cdot \mathbf{r}_{1}(\mathbf{x})}{\left|\mathbf{r}_{1}(\mathbf{x})\right|} \\
f_{3}= & H_{0}^{(1)}\left(\kappa_{1}\left|\mathbf{r}_{4}(\mathbf{x})\right|\right)-H_{0}^{(1)}\left(\kappa_{0}\left|\mathbf{r}_{2}(\mathbf{x})\right|\right) \\
f_{4}= & -\widetilde{\epsilon}_{1} \omega \kappa_{1} H_{1}^{(1)}\left(\kappa_{1}\left|\mathbf{r}_{3}(\mathbf{x})\right|\right) \frac{\widehat{\boldsymbol{n}}(\mathbf{x}) \cdot \mathbf{r}_{3}(\mathbf{x})}{\left|\mathbf{r}_{3}(\mathbf{x})\right|} \\
& +\beta_{1} \kappa_{1} H_{1}^{(1)}\left(\kappa_{1}\left|\mathbf{r}_{4}(\mathbf{x})\right|\right) \frac{\widehat{\boldsymbol{\tau}}(\mathbf{x}) \cdot \mathbf{r}_{4}(\mathbf{x})}{\left|\mathbf{r}_{4}(\mathbf{x})\right|} \\
& +\widetilde{\epsilon}_{0} \omega \kappa_{0} H_{1}^{(1)}\left(\kappa_{0}\left|\mathbf{r}_{1}(\mathbf{x})\right|\right) \frac{\widehat{\boldsymbol{n}}(\mathbf{x}) \cdot \mathbf{r}_{1}(\mathbf{x})}{\left|\mathbf{r}_{1}(\mathbf{x})\right|} \\
& -\beta_{0} \kappa_{0} H_{1}^{(1)}\left(\kappa_{0}\left|\mathbf{r}_{2}(\mathbf{x})\right|\right) \frac{\widehat{\boldsymbol{\tau}}(\mathbf{x}) \cdot \mathbf{r}_{2}(\mathbf{x})}{\left|\mathbf{r}_{2}(\mathbf{x})\right|}
\end{aligned}
$$

where $\mathbf{r}_{k}(\mathbf{x})=\mathbf{x}-\mathbf{z}_{k}$. Then, the fields

$$
\begin{aligned}
& u_{0}(\mathbf{x})=H_{0}^{(1)}\left(\kappa_{0}\left|\mathbf{x}-\mathbf{z}_{1}\right|\right), \quad v_{0}(\mathbf{x})=H_{0}^{(1)}\left(\kappa_{0}\left|\mathbf{x}-\mathbf{z}_{2}\right|\right), \quad \mathbf{x} \in \Omega_{0}, \\
& u_{1}(\mathbf{x})=H_{0}^{(1)}\left(\kappa_{1}\left|\mathbf{x}-\mathbf{z}_{3}\right|\right), \quad v_{1}(\mathbf{x})=H_{0}^{(1)}\left(\kappa_{1}\left|\mathbf{x}-\mathbf{z}_{4}\right|\right), \quad \mathbf{x} \in \Omega_{1},
\end{aligned}
$$

solve the following problem

$$
\begin{array}{lll}
\Delta u_{0}+\kappa_{0}^{2} u_{0}=0, & \Delta v_{0}+\kappa_{0}^{2} v_{0}=0, & \mathbf{x} \in \Omega_{0}, \\
\Delta u_{1}+\kappa_{1}^{2} u_{1}=0, & \Delta v_{1}+\kappa_{1}^{2} v_{1}=0, & \mathbf{x} \in \Omega_{1},
\end{array}
$$

with boundary conditions

$$
\begin{aligned}
u_{1} & =u_{0}+f_{1}, & & \mathbf{x} \in \Gamma, \\
\widetilde{\mu}_{1} \omega \frac{\partial v_{1}}{\partial n}+\beta_{1} \frac{\partial u_{1}}{\partial \tau} & =\widetilde{\mu}_{0} \omega \frac{\partial v_{0}}{\partial n}+\beta_{0} \frac{\partial u_{0}}{\partial \tau}+f_{2}, & & \mathbf{x} \in \Gamma, \\
v_{1} & =v_{0}+f_{3}, & & \mathbf{x} \in \Gamma, \\
\widetilde{\epsilon}_{1} \omega \frac{\partial u_{1}}{\partial n}-\beta_{1} \frac{\partial v_{1}}{\partial \tau} & =\widetilde{\epsilon}_{0} \omega \frac{\partial u_{0}}{\partial n}-\beta_{0} \frac{\partial v_{0}}{\partial \tau}+f_{4}, & & \mathbf{x} \in \Gamma,
\end{aligned}
$$




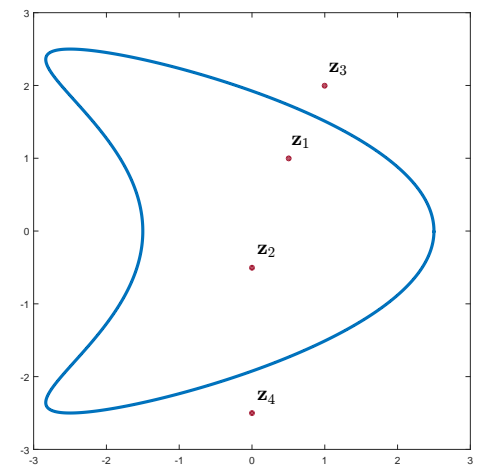

FIgURE 2. The parametrization of the boundary $\Gamma$ and the source points.

and the radiation conditions

$$
\lim _{r \rightarrow \infty} \sqrt{r}\left(\frac{\partial u_{0}}{\partial r}-i \kappa_{0} u_{0}\right)=0, \quad \lim _{r \rightarrow \infty} \sqrt{r}\left(\frac{\partial v_{0}}{\partial r}-i \kappa_{0} v_{0}\right)=0 .
$$

For this problem, we can again derive a system as (3.14), where now $\mathbf{b}$ is replaced by

$$
\mathbf{b}_{f}=\left(\begin{array}{c}
-\left(1 / \widetilde{\epsilon}_{0} \omega\right) S_{0} f_{4}+\left(\widetilde{\epsilon}_{1} / \widetilde{\epsilon}_{0}\right) S_{0} K_{1} f_{1}+\left(\beta_{1} / \widetilde{\epsilon}_{0} \omega\right) S_{0} L_{1} f_{3} \\
-\left(1 / \widetilde{\mu}_{0} \omega\right) S_{0} f_{2}+\left(\widetilde{\mu}_{1} / \widetilde{\mu}_{0}\right) S_{0} K_{1} f_{3}-\left(\beta_{1} / \widetilde{\mu}\right)_{0} \omega S_{0} L_{1} f_{1}
\end{array}\right)
$$

Given (4.4) and the asymptotic behavior of the Hankel function [4], we know that the far field patterns of $u_{0}$ and $v_{0}$ are given by

$$
\begin{array}{ll}
u_{0}^{\infty}(\widehat{\boldsymbol{x}})=\frac{-4 i e^{i \pi / 4}}{\sqrt{8 \pi \kappa_{0}}} e^{-i \kappa_{0} \widehat{\boldsymbol{x}} \cdot \mathbf{z}_{1}}, \quad \widehat{\boldsymbol{x}} \in S, \\
v_{0}^{\infty}(\widehat{\boldsymbol{x}})=\frac{-4 i e^{i \pi / 4}}{\sqrt{8 \pi \kappa_{0}}} e^{-i \kappa_{0} \widehat{\boldsymbol{x}} \cdot \mathbf{z}_{2}}, \quad \widehat{\boldsymbol{x}} \in S,
\end{array}
$$

where $S$ is the unit ball. Numerically, the far field patterns are given 

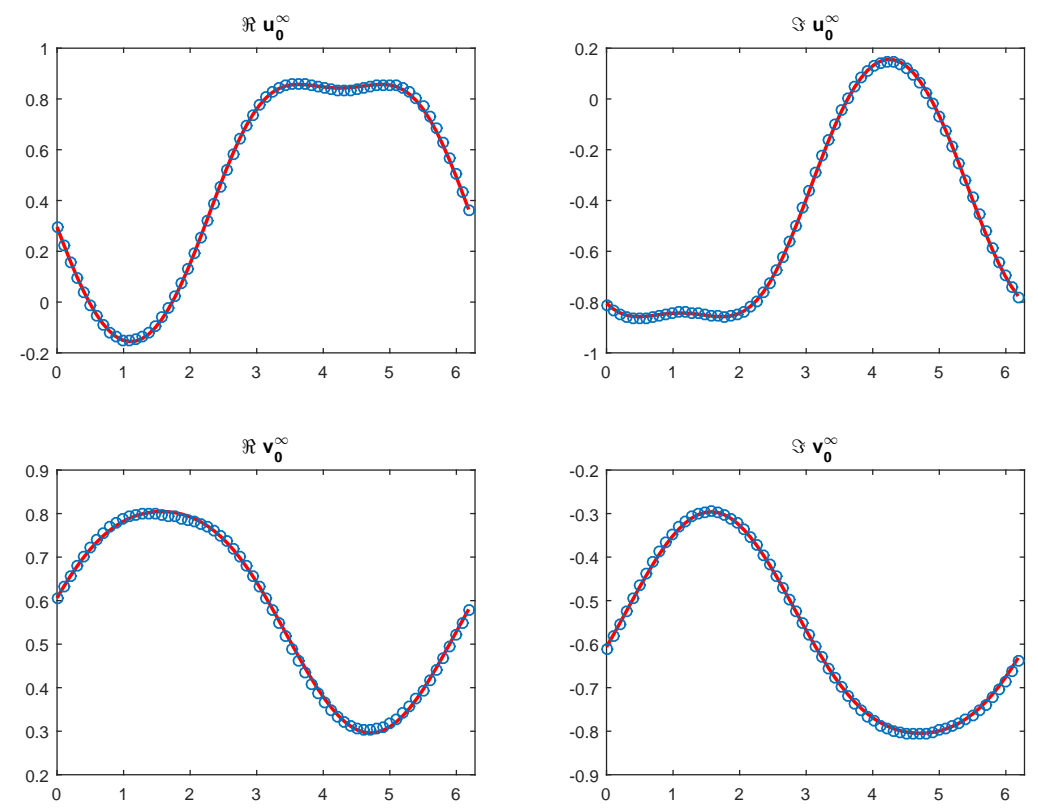

Figure 3. The far field patterns: Reconstructed (blue open circles) and exact (red solid line).

by

$$
\begin{aligned}
u_{0}^{\infty}(\widehat{\boldsymbol{x}}) & =\frac{e^{i \pi / 4}}{\sqrt{8 \pi \kappa_{0}}} \int_{0}^{2 \pi} e^{-i \kappa_{0} \widehat{\boldsymbol{x}} \cdot \mathbf{z}(s)}\left[-i \kappa_{0}(\widehat{\boldsymbol{x}} \cdot \widehat{\boldsymbol{n}}(s)) \varphi_{0}(s)\right. \\
& \left.\left.-\left(K_{0} \varphi_{0}\right)(s)\right]\left|\mathbf{z}^{\prime}(s)\right|\right] d s \\
v_{0}^{\infty}(\widehat{\boldsymbol{x}}) & =\frac{e^{i \pi / 4}}{\sqrt{8 \pi \kappa_{0}}} \int_{0}^{2 \pi} e^{-i \kappa_{0} \widehat{\boldsymbol{x}} \cdot \mathbf{z}(s)}\left[-i \kappa_{0}(\widehat{\boldsymbol{x}} \cdot \widehat{\boldsymbol{n}}(s)) \psi_{0}(s)\right. \\
& \left.-\left(K_{0} \psi_{0}\right)(s)\right]\left|\mathbf{z}^{\prime}(s)\right| d s
\end{aligned}
$$

where $\boldsymbol{\varphi}:=\left(\varphi_{0}, \psi_{0}\right)^{T}$ solves

$$
(\mathbf{D}+\mathbf{K}) \varphi=\mathbf{b}_{f}
$$




\begin{tabular}{|c|c|c|c|c|c|c|}
\cline { 2 - 7 } \multicolumn{1}{c|}{} & \multicolumn{3}{|c|}{$u_{0}^{\infty}$} & \multicolumn{3}{c|}{$v_{0}^{\infty}$} \\
\hline$n t$ & 0 & $\pi / 2$ & $\pi$ & 0 & $\pi / 2$ & $\pi$ \\
\hline 32 & 0.006470 & 0.009056 & 0.003944 & 0.004714 & 0.007460 & 0.001767 \\
\hline 64 & 0.003157 & 0.004729 & 0.001820 & 0.002249 & 0.004288 & 0.000601 \\
\hline 128 & 0.001581 & 0.002374 & 0.000908 & 0.001125 & 0.002161 & 0.000292 \\
\hline
\end{tabular}

TABLE 1. Absolute errors of the far field patterns of $u_{0}$ and $v_{0}$ for different orders $n$ at discrete points $t$.

Here, we have used the representations (3.10) for the exterior fields and the asymptotics of the Hankel function. The operator $K_{0}$ is given by (3.13).

We consider the points $\mathbf{z}_{1}=(0.5,1)$ and $\mathbf{z}_{2}=(0,-0.5)$ in $\Omega_{1}$ and the points $\mathbf{z}_{3}=(1,2)$ and $\mathbf{z}_{4}=(0,-2.5)$ in $\Omega_{0}$, see Figure 2 . We set $\omega=1$ and $n=32$. The exact values (4.5) and the reconstructed (4.6) for $\left(\epsilon_{1}, \mu_{1}\right)=(3,2)$ and $\left(\epsilon_{0}, \mu_{0}\right)=(1,1)$ are presented in Figure 3. The results are presented for $\theta=\pi / 3$. In Table 1 , we provide the absolute errors of the far field patterns for different values of $n$ and $t$.

As a general comment, we could say that the reconstructions are accurate and illustrate the feasibility of the proposed method. However, the convergence is slower compared to the impedance cylinder case [23]. The main reason is the complexity of the matrix $\mathbf{K}$ involving the product of four operators: $S_{0} T S_{j}\left(N S_{j}+1 / 2 I\right)^{-1} N D_{j}, j=0,1$ resulting in an increase of the condition number. As $\theta \rightarrow \pi / 2$, the results improve considerably.

4.2. Example with oblique incidence. In this example, we consider the usual obliquely incident (TM) polarized electromagnetic plane wave, resulting in the forms (2.10). We keep the same values for all parameters as in the previous example. We restrict the computations of the fields to the rectangular domain $[-5,5]^{2}$, and we consider a two-dimensional uniform-space discretization, namely, $\mathbf{x}_{k j}=$ $(-5+k \delta,-5+j \delta)$, where $\delta=10 /(2 m-1)$, for $k, j=0, \ldots, 2 m-1$. We use $m=128$. 

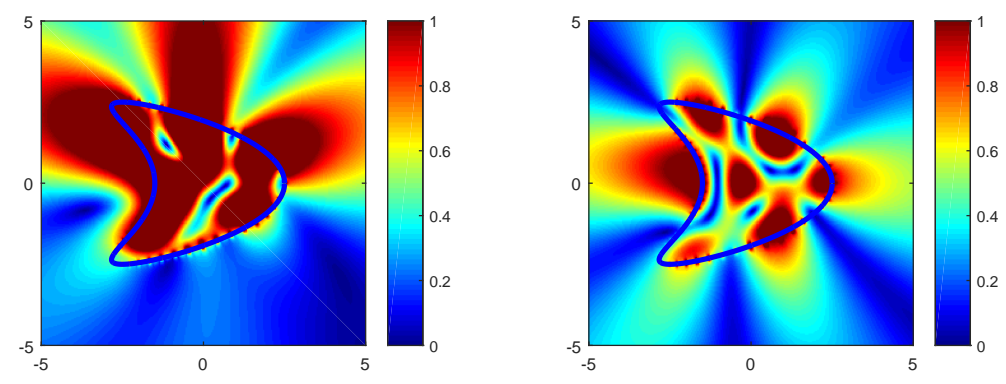

FiguRE 4. The norms of the electric fields $u_{0}$ and $u_{1}$ (left) and of the magnetic fields $v_{0}$ and $v_{1}$ (right) for $\phi=\pi / 2$.
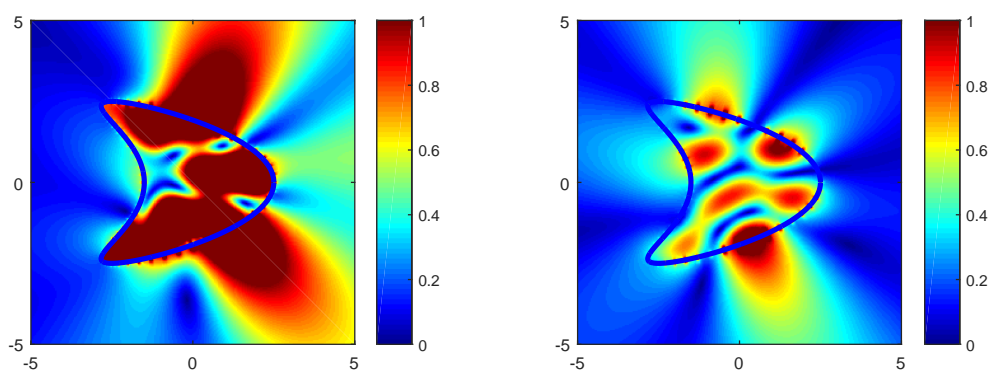

FigurE 5 . The norms of the electric fields $u_{0}$ and $u_{1}$ (left) and of the magnetic fields $v_{0}$ and $v_{1}$ (right) for $\phi=\pi / 9$.

The values of the norms of the scattered electric and magnetic fields $\left|u_{0}\right|,\left|v_{0}\right|$ and the interior electric and magnetic fields $\left|u_{1}\right|,\left|v_{1}\right|$ are presented in Figures 4 and 5 for different values of the polar angle $\phi$, which in $\mathbb{R}^{2}$ corresponds to the incident direction $(\cos \phi, \sin \phi) \in S$.

Acknowledgments. The authors thank the referees for their valuable comments. This research was initiated while DG was visiting the Department of Mathematical Sciences, University of Delaware and he expresses his gratitude for its hospitality. 


\section{REFERENCES}

1. F. Cakoni and D. Colton, Qualitative methods in inverse scattering theory, Springer-Verlag, Berlin, 2006.

2. A.C. Cangellaris and R. Lee, Finite element analysis of electromagnetic scattering from inhomogeneous cylinders at oblique incidence, IEEE Trans. Ant. Prop. 39 (1991), 645-650.

3. D. Colton and R. Kress, Integral equation methods in scattering theory, Pure Appl. Math., John Wiley \& Sons Inc., New York, 1983.

4. Inverse acoustic and electromagnetic scattering theory, 2nd edition, Appl. Math. Sci. 93, Springer-Verlag, Berlin, 1998.

5. M. Costabel and E. Stephan, A direct boundary integral equation method for transmission problems, J. Math. Anal. Appl. 106 (1985), 367-413.

6. R. Courant and D. Hilbert, Methods of mathematical physics, volume 2, Wiley-Interscience, New York, 1962.

7. V. Isakov, Inverse problems for partial differential equations, Appl. Math. Sci. 127, Springer, New York, 2006.

8. R. Kittappa and R.E. Kleinman, Acoustic scattering by penetrable homogeneous objects, J. Math. Phys. 16 (1975), 421-432.

9. R.E. Kleinman and P.A. Martin, On single integral equations for the transmission problem of acoustics, SIAM J. Appl. Math. 48 (1988), 307-325.

10. R. Kress, On the numerical solution of a hypersingular integral equation in scattering theory, J. Comp. Appl. Math. 61 (1995), 345-360.

11. , Linear integral equations, 2nd edition, Springer Verlag, Berlin, 1999.

12. A collocation method for a hypersingular boundary integral equation via trigonometric differentiation, J. Integral Equations Appl. 26 (2014), 197213.

13. M. Lucido, G. Panariello and F. Schettiho, Scattering by polygonal crosssection dielectric cylinders at oblique incidence, IEEE Trans. Ant. Prop. 58 (2010), $540-551$.

14. A.W. Maue, Über die formulierung eines allgemeinen beugungsproblems durch eine integralgleichung, Z. Phys. 126 (1949), 601-618.

15. K.M. Mitzner, Acoustic scattering from an interface between media of greatly different density, J. Math. Phys. 7 (1966), 2053-2060.

16. P. Monk, Finite element methods for maxwells equations, Oxford University Press, Oxford, 2003.

17. G. Nakamura and H. Wang, The direct electromagnetic scattering problem from an imperfectly conducting cylinder at oblique incidence, J. Math. Anal. Appl. 397 (2013), 142-155.

18. J.C. Nédélec, Acoustic and electromagnetic equations, Springer-Verlag, New York, 2001. 
19. R.G. Rojas, Scattering by an inhomogeneous dielectric/ferrite cylinder of arbitrary cross-section shape-oblique incidence case, IEEE Trans. Ant. Prop. 36 (1988), 238-246.

20. J.L. Tsalamengas, Exponentially converging nyström methods applied to the integral-integrodifferential equations of oblique scattering/hybrid wave propagation in presence of composite dielectric cylinders of arbitrary cross section, IEEE Trans. Ant. Prop. 55 (2007), 3239-3250.

21. N.L. Tsitsas, E.G. Alivizatos, H.T. Anastassiu and D.I. Kaklamani, Optimization of the method of auxiliary sources (mas) for oblique incidence scattering by an infinite dielectric cylinder, Electr. Engin. 89 (2007), 353-361.

22. J.R. Wait, Scattering of a plane wave from a circular dielectric cylinder at oblique incidence, Canadian J. Phys. 33 (1955), 189-195.

23. H. Wang and G. Nakamura, The integral equation method for electromagnetic scattering problem at oblique incidence, Appl. Num. Math. 62 (2012), 860873 .

24. J. Yan, R.K. Gordon and A.A. Kishk, Electromagnetic scattering from impedance elliptic cylinders using finite difference method, Electromagnetism $\mathbf{1 5}$ (1995), 157-173.

25. H.A. Yousif and A.Z. Elsherbeni, Oblique incidence scattering from two eccentric cylinders, J. Electr. Waves Appl. 11 (1997), 1273-1288.

Department of Mathematics, National Technical University of Athens, GreEce

Email address: dgindi@math.ntua.gr

Computational Science Center, University of Vienna, Austria

Email address: leonidas.mindrinos@univie.ac.at 